KANAZAWA-13-09, HUPD-1308, KEK-CP-290

\title{
Lattice Study on quantum-mechanical dynamics of two-color QCD with six light flavors
}

\author{
M. Hayakawa, ${ }^{1}$ K.-I. Ishikawa, ${ }^{2}$ S. Takeda, ${ }^{3}$ M. Tomii, ${ }^{1}$ and N. Yamada ${ }^{4,5}$ \\ ${ }^{1}$ Department of Physics, Nagoya University, Nagoya 464-8602, Japan \\ ${ }^{2}$ Department of Physics, Hiroshima University, \\ Higashi-Hiroshima, 739-8526, Japan \\ ${ }^{3}$ School of Mathematics and Physics, \\ College of Science and Engineering, \\ Kanazawa University, Kakuma-machi, \\ Kanazawa, Ishikawa 920-1192, Japan \\ 4 KEK Theory Center, Institute of Particle and Nuclear Studies, \\ High Energy Accelerator Research Organization (KEK), Tsukuba 305-0801, Japan \\ 5 School of High Energy Accelerator Science, \\ Graduate University for Advanced Studies (Sokendai), Tsukuba 305-0801, Japan
}

(Dated: September 13, 2018)

\begin{abstract}
We investigate the chiral properties of $\mathrm{SU}(2)_{\mathrm{C}}$ gauge theory with six flavors, i.e. six light Dirac fermions in the fundamental representations by lattice simulation, and point out that the spontaneous breakdown of chiral symmetry does not occur in this system. The quark mass dependence of the mesonic spectrum provides an evidence for such a possibility. The decay constant tends to be increased by the finite size effect, which is opposite to the behavior predicted by chiral perturbation theory and indicates that the long distance dynamics in the six-flavor theory could be different from the theory with chiral symmetry breaking. The subtracted chiral condensate, whose utility is demonstrated by the simulation of two-flavor theory, is shown to vanish in the chiral limit within the precision of available data.
\end{abstract}

PACS numbers: 11.15.-q,11.15.Ha,12.38.Gc 


\section{INTRODUCTION}

The standard model contains a Higgs doublet field which plays the role to trigger the electroweak symmetry breakdown and to generate the masses of all known elementary particles. The standard model, however, is incapable of predicting observed flavor structure; all of fermion masses as well as mixing angles require corresponding number of input parameters. The technicolor [1, 2] and its extension [3, 4] are such an attempt to attribute flavor structure and breakdown of electroweak symmetry to quantum-mechanical dynamics of some gauge theory. The components absorbed into $\mathrm{W}$ and $\mathrm{Z}$ bosons eventually resolve into their constituent fermions at shorter distance. Therefore, stability of electroweak scale against radiative correction is guaranteed.

However, the technicolor dynamics must be quite different from QCD to resolve inconsistency with the size of flavor changing neutral current (See, for example, Ref. [5]) and the constraints on the nature of electroweak symmetry breakdown [6, 7]. The former problem can be solved if technicolor dynamics give rise to relatively large anomalous dimension for the chiral condensate over wide range of energy scale (walking technicolor) [8 [11]. It needs, however, explicit nonperturbative calculation in order to access to the question whether such dynamics settle the second problem. The simulation formulated on lattice gauge theory is expected to play a vital role to answer to this question [12 14], once we successfully find out the gauge system with walking dynamics.

Fairly many works have been done to search a candidate for walking technicolor by means of lattice simulation after the seminal work [15, 16], which gave an evidence for the conformality in the infrared (IR) limit of $\mathrm{SU}(3)_{\mathrm{C}}$ gauge theory with twelve light Dirac fermions by calculating the running gauge coupling constant defined in the Schrödinger functional scheme (SF) [17 19]. Other than SF coupling constant calculation, various methods have been proposed and attempted to approach the issue; analysis of phase structure of lattice systems [20, 21], finite size scaling test [22], large scale spectroscopy study [23], Monte-Carlo renormalization group approach [24 26], and test of hyperscaling relations in the attractive basin of infrared fixed point (IRFP) [27, 28], the calculation of coupling constant defined via twisted Polyakov loop [29, 30], and the search of modified asymptotic behavior of the correlation function [31].

Thus far, $\mathrm{SU}(3)_{\mathrm{C}}$ gauge theories have been most often investigated as a candidate gauge 
system, in particular, with fermions in the fundamental representations, called flavors. In a series of such $N_{F}$-flavor QCD, the work [32] showed that ten-flavor QCD may have large mass anomalous dimension $\gamma_{m} \sim 1$ at the IRFP, the existence of which was demonstrated by the SF coupling calculation [33].

We focus here on a series of $\mathrm{SU}(2)_{\mathrm{C}}$ gauge theories to search a candidate gauge theory with walking technicolor dynamics. It is probable that the quantum dynamics of $\mathrm{SU}(2)_{\mathrm{C}}$ gauge theories differ from those of $\mathrm{SU}(3)_{\mathrm{C}}$ gauge theories. Firstly, in pure $\mathrm{SU}(2)_{\mathrm{C}}$ Yang-Mills theory, the finite temperature transition from confinement to deconfinement is second-order [34, 35] and belongs to the universality class of three-dimensional Ising model [36], while it is first-order in pure $\mathrm{SU}(3)_{\mathrm{C}}$ Yang-Mills theory. If $\mathrm{SU}(2)_{\mathrm{C}}$ gauge theory involves fermions coupled to the gauge field, it should not be regarded as one of $\mathrm{SU}\left(N_{C}\right)_{\mathrm{C}}$ gauge theories; rather it is one of $\operatorname{Sp}(2 N)_{\mathrm{C}}$ gauge theories, in which the fundamental representation is pseudoreal. As a consequence, $\mathrm{SU}(2)_{\mathrm{C}}$ gauge theory with $N_{F}$ Dirac fermions in the fundamental representation, often referred to as two-color QCD with $N_{F}$-flavors of "quarks", has the chiral symmetry $\mathrm{SU}\left(2 N_{F}\right)$ enhanced from the familiar $\mathrm{SU}\left(N_{F}\right)_{\mathrm{L}} \times \mathrm{SU}\left(N_{F}\right)_{\mathrm{R}} \times \mathrm{U}(1)_{\mathrm{B}}$. (Appendix A summarizes this point.) It is thus anticipated that the chiral dynamics of two-color QCD can differ significantly from those of three-color QCD, in particular, at the critical number $N_{F}^{\text {crtl }}$ of fermions, above which gauge system becomes conformal in the infrared limit, called IR-conformal.

Moreover, from the point of view of the application to the dynamical realization of the electroweak symmetry breaking, the content of the effective Higgs sector is quite different in $\mathrm{Sp}(2 N)_{\mathrm{C}}$ gauge theories from that in $\mathrm{SU}\left(N_{C}\right)_{\mathrm{C}}\left(N_{C} \geq 3\right)$, as described in Appendix A. This fact also motivates us to carry out lattice simulation to grasp its properties of nonperturbative dynamics such as spectra of bound states.

Study of two-color QCD intended to search the conformal window has been done by means of lattice simulation so far $[20,37-42]$. The perturbatively calculated $\beta$ function [43] suggests that $6 \leq N_{F}^{\text {crtl }} \leq 8$. Ref. [20] investigated the phase structure of Wilson fermions and indicated that $N_{F}^{\text {crtl }}=2$ and that the massless theory is not in the confinement phase for $N_{F} \geq 3$. Ref. [38] is the first trial to compute the SF running gauge coupling constant for the six-flavor theory, and pointed out the existence of infrared fixed point (IRFP) in the region $4 \lesssim g_{\star}^{2} \lesssim 6$. The authors in Ref. [40] use $O(a)$-improved fermionic actions (clover actions) and find no evidence of IRFP at least in the region $g_{\star}^{2} \lesssim 9$. Recently, Ref. [42] suggests the 
absence of IRFP through the calculation of the SF running coupling with smeared link fields, where the continuum limit has not been taken yet. Our latest result for the SF coupling constant with perturbative improvement indicates that IR fixed point $g_{\star}^{2}$ exists in the range [44]

$0.06<\frac{1}{g_{\star}^{2}}<0.15$.

The purpose of this paper is to report our result for chiral properties of $\mathrm{SU}(2)_{\mathrm{C}}$ gauge theory with six Dirac fermions in the fundamental representation by the lattice simulation. Overall, our strategy here is complementary to the calculation of the SF running coupling constant reported in a separate paper [44]. The SF running coupling constant is obtained by taking the explicit continuum limit of the data for the massless quark. Instead, we focus here on the dependence on the quark mass of the various observables, such as mesonic spectra, decay constant, etc. In the report [45] on the preliminary result, the gluonic observables are analyzed to investigate their fate in the chiral limit, but we leave them for the future study until further accumulation of statistics is accomplished. In this paper, we concentrate on the investigation of the chiral properties of two-color QCD with the six-flavors, which has not been done in Ref. [45].

Here we attempt to get a new insight on the possible role of the finite size effect so that it can provide valuable knowledge concerning with the long distance properties of quantummechanical dynamics (Sec. IIE). We are inspired by the result for the mesonic and gluonic spectra in the $\mathrm{SU}(2)_{\mathrm{C}}$ gauge theory with two fermions in the adjoint representation obtained in Ref. [27]. In three-color QCD with two-flavors, the finite size effect is known to increase the mass of, say, the lightest pseudoscalar meson, pion [46]. However, in the $\mathrm{SU}(2)_{\mathrm{C}}$ gauge theory with two adjoint fermions, the pion mass is decreased by the finite size effect. Another notable result found in Ref. [27] is the fact that the $0^{++}$glueball is lighter than the pion in the measured quark mass range, which is in striking contrast to the usual QCD. This tendency is actually compatible with the theory of the finite size effect on the meson masses [47 49]. In Sec. IE, we focus on the decay constant, and list possible tendency for the finite size effects in order to compare them with our data. We find that $f_{P}$ is decreased by the finite size effect in the six-flavor theory, which is opposite to the behavior predicted by the chiral perturbation theory.

Our results for the meson masses and the decay constant $f_{P}$ in the six-flavor system 
have a similarity with those found for $\mathrm{SU}(3)_{\mathrm{C}}$ gauge theory with two Dirac fermions in the symmetric representation [21]. A look at the formula to calculate $f_{P}$, Eq. (9), indicates that $f_{P}$ approaches to zero in the chiral limit once the pion mass is bounded below. In Sec. III, we make a preliminary study of finite size effects in the theory with chiral symmetry breaking at very weak coupling, as it may provide a benchmark to decide if the observed behavior is caused by the genuine dynamics of the IR-conformal theory or just due to the finite size effect.

The paper is organized as follows. Section II describes the method of calculation. There, we also discuss the possible role of finite size effects to discriminate between the theory with chiral symmetry breaking and the IR-conformal theory. Section. III has two purposes. One is to demonstrate the utility of the subtracted chiral condensate defined through the pseudoscalar correlator (See Eq. (11)). Another is to get the knowledge on the finite size

effects on the various observables in the theory with chiral symmetry breaking, but at such weak coupling that the chiral perturbation theory at finite volume is not applicable. Those knowledges will be confronted with the data to be obtained for our target system, $N_{F}=6$ theory. After explaining the choice for the value of the bare coupling constant in Sec. IV] we present our results in Sec. V. Section VI is devoted to the summary of this paper and discussion.

\section{LATTICE SIMULATION}

\section{A. generation of gauge configurations}

To study $\mathrm{SU}(2)_{\mathrm{C}}$ gauge theory with six dynamical Dirac fermions, it is necessary to start with generating a certain finite number of gauge configurations of this system. They form a sample to estimate the ensemble average corresponding to the vacuum expectation value $(\mathrm{VEV})\langle\mathcal{A}\rangle$ of an operator, $\mathcal{A}$, with respect to some lattice-regularized Euclidean action

$S_{\text {lat }}=S_{G}+S_{F}$,

or the secondary quantities.

In the lattice action (2), $S_{G}$ is given solely by the gauge field, so called link variable $U(n, \mu)$ obeying periodic boundary condition. In this paper, we employ the plaquette gauge 
action for

$$
\begin{aligned}
S_{\mathrm{G}}[U] & =\frac{\beta}{2 N_{C}} \sum_{n \in \Gamma_{4}} \sum_{0 \leq \mu<\nu \leq 3} 2 \operatorname{Retr}(1-U(n ; \mu, \nu)), \\
U(n ; \mu, \nu) & \equiv U(n, \mu) U(x+\widehat{\mu}, \nu) U(n+\widehat{\nu}, \mu)^{-1} U(n, \nu)^{-1},
\end{aligned}
$$

where $\widehat{\mu}$ denotes the unit vector along the $\mu$-direction, and the sum is taken over the whole lattice points (sites) $\Gamma_{4}$.

$S_{F}$ in Eq. (2) is the part containing the coupling of the "quarks" $\psi_{j}, \bar{\psi}_{j}$ to the gauge field. In this paper, we employ the Wilson fermion action for $S_{F}$

$$
\begin{aligned}
S_{F}[\psi, \bar{\psi}, U]= & \sum_{n \in \Gamma_{4}} \sum_{j=1}^{N_{F}} \bar{\psi}_{j}(n)\left(D_{W}[U] \psi_{j}\right)(n) \\
= & \sum_{n \in \Gamma_{4}} \sum_{j=1}^{N_{F}}\left[\begin{array}{r}
\bar{\psi}_{j}(n) \psi_{j}(n)-\kappa \sum_{\mu=1}^{4}\left\{\bar{\psi}_{j}(n)\left(1-\gamma_{\mu}\right) U(n, \widehat{\mu}) \psi_{j}(n+\widehat{\mu})\right. \\
\left.\left.+\bar{\psi}_{j}(n+\widehat{\mu})\left(1+\gamma_{\mu}\right) U(n, \widehat{\mu})^{\dagger} \psi_{j}(n)\right\}\right] .
\end{array}\right.
\end{aligned}
$$

The Wilson fermion action $S_{F}$ contains a parameter $\kappa$, called hopping parameter, representing the strength of the nearest-neighboring fermionic variables, in place of the bare quark mass. In the simulation, we adopt the periodic boundary conditions for $\psi, \bar{\psi}$ along all directions. Actually, in the quantum $\mathrm{SU}(2)_{C}$ gauge theory, the anti-periodic boundary condition is easily seen to be equivalent to the periodic boundary condition if all the representation of the matter fields are the $Z_{2}$-odd conjugacy classes, such as fundamental representation.

The gauge configurations corresponding to the action (2) are produced in the standard manner. The important sampling is processed according to the lattice action (2) by Hybrid Monte-Carlo method (HMC) [50], which utilizes $N_{F} / 2\left(=3\right.$ for $\left.N_{F}=6\right)$ pseudo-fermions to represent $N_{F}$ copies of functional determinants of the Wilson Dirac operator $D_{W}[U]$. The evolution in the modular dynamics is numerically performed by the improved integrator [51] . The most computationally expensive part occupied in this evolution and the calculation of change of stochastic Hamiltonian is solving the linear problem, $D_{W}[U] \phi=b$. This part can be accelerated by using 3 GPU cards and adopting the mixed precision solver with flavor-parallelized single-precision preconditioning [52]. 


\section{B. meson masses}

Throughout this paper, a "meson" means a color-singlet and flavor-nonsinglet state with vanishing baryon number. In the rest of this section, all quantities such as meson masses are dimensionless. The masses of the lightest mesons in the channel $H \in\{\mathrm{P}, \mathrm{V}, \mathrm{S}, \mathrm{A}\}$ are determined by measuring the connected-type contribution to the two-point function

$f_{H H}\left(t \equiv n_{0}\right) \equiv \sum_{\mathbf{n}}\left\langle O_{H}(n) O_{H}(0)^{\dagger}\right\rangle$.

$O_{H}(n)$ is a certain bilinear field with the quantum number corresponding to $H$, for instance, the flavor-non-singlet and strictly local operator $O_{P}(n)=P(n) \equiv \bar{\psi}(n) \gamma_{5} \psi^{\prime}(n)$ in the pseudoscalar channel $P$. Unless the fermions are massless, the excited state gets non-zero energy gap from the ground state in every channel even in the IR-conformal theory. The mass $M_{H}$ of the ground state is extracted from the asymptotic behavior of the correlation function (5) at large $t$ so that the contribution of the excited states all drop out and it is dominated by the ground state contribution

$f_{H H}(t)=A^{H H} e^{-M_{H} t}+\cdots$.

The one-pole dominance can be monitored by checking if the effective mass

$M_{H H}(t) \equiv \ln \left(\frac{f_{H H}(t)}{f_{H H}(t+1)}\right)$,

exhibits a plateau. Actually, there are channels $H$ on the lattices which do not exhibit plateau, probably due to short length along the time direction. We then give up finding $M_{H}$. If something like plateau is seen in a certain limited range of time, the correlation function is measured with the gauge-invariant smeared source as in Ref. [53] (Jacobi smearing) for the operator $O_{H}(0)$. The corresponding effective mass $M_{H H}(t)$ approaches to a plateau from below, enabling to check if its position is consistent with that observed using the point source.

\section{PCAC mass, decay constant}

The Wilson fermion explicitly breaks chiral symmetry. Thus, the "naive mass" proportional to $1 / \kappa$ suffers additive and nonperturbative shift. Instead, mimicking the PCAC 
relation in the continuum theory, (bare) PCAC mass $m_{\mathrm{PCAC}}$ derived from the plateau of the ratio of the correlation functions summed over spatial volume

$$
\begin{aligned}
\widehat{m}_{\mathrm{PCAC}}(t) & \equiv \frac{\frac{1}{2}\left(\partial_{t}+\partial_{t}^{*}\right) f_{A_{t} P}(t)}{2 f_{P P}(t)}, \\
f_{A_{t} P}\left(t \equiv n_{0}\right) & \equiv \sum_{\vec{n}}\left\langle A_{t}(n) P(0)\right\rangle,
\end{aligned}
$$

where $A_{t}(n) \equiv \bar{\psi}^{\prime} \gamma_{t} \gamma_{5} \psi$ and $\partial_{t}\left(\partial_{t}^{*}\right)$ is the forward (backward) difference operator, is used as a measure of bare quark mass.

Similarly, the (bare) decay constant $f_{P}$ of the lightest pseudoscalar meson is obtained as $f_{P}=(2 \kappa) 2 m_{\mathrm{PCAC}} \sqrt{\frac{2 A^{P P}}{M_{P}}} \frac{1}{\sinh \left(M_{P}\right)}$.

In the context of the application to the electroweak symmetry breaking, the decay constant in the continuum theory serves the mass scale to make qualitative predictions, and will be obtained by multiplying the above $f_{P}$ by the constant $Z_{A}$ of the renormalization of the axial-vector current and an appropriate matching factor. Since our main interest here is to explore the qualitative features of the dynamics of the system, the multiplication of all renormalization factors, which are short-distance quantities and do not affect to the long distance dynamics, is omitted.

\section{D. subtracted chiral condensate}

The Wilson fermion breaks chiral symmetry explicitly through the Wilson term. As a consequence, the vacuum expectation value of the chiral condensate $\langle\bar{\psi}(0) \psi(0)\rangle$ (no sum over flavors is understood throughout this paper for this quantity) suffer hard ultra-violet divergence $\sim O\left(a^{-3}\right)$, the singularity that does not vanish in the massless quark limit. The renormalization requires the subtraction of such a hard component as the first step. We will call the resulting chiral condensate as the subtracted chiral condensate. It is possible to calculate

$$
\langle\bar{\psi}(0) \psi(0)\rangle=-\left\langle\operatorname{tr}^{\prime} D_{W}[U]^{-1}(0)\right\rangle,
$$

where $D_{W}[U]^{-1}$ is the inverse of the Wilson Dirac operator in Eq. (4) and $\operatorname{tr}^{\prime}$ implies the sum over colors. However, no one knows a practical method to subtract away such an $O\left(a^{-3}\right)$ component to get the subtracted chiral condensate. 
Even for the Wilson fermion, the Ward-Takahashi identity with respect to the axial-vector current can be written down [54]

$\delta^{a b} \cdot\langle\bar{\psi} \psi\rangle_{\text {subt }}\left(m_{\mathrm{PCAC}}, L / a\right)=2 m_{\mathrm{PCAC}} \cdot(2 \kappa)^{2} \sum_{n}\left\langle P^{a}(n) P^{b}(0)\right\rangle$

where $P^{a}(n)=\bar{\psi} T^{a} \gamma_{5} \psi$ for $\mathrm{SU}\left(N_{F}\right)$ generators $T^{a}$. Through this identity, the subtracted chiral condensate in the Wilson fermion is calculated using the right hand side of Eq. (111). The quantity in Eq. (11) actually requires the multiplicative renormalization constants to be confronted with the continuum physics. Those constants are short-distance quantities, and are thus considered not to affect to the observation on the essence of the long distance dynamics of the system.

Recently, $\langle\bar{\psi} \psi\rangle_{\text {subt }}$ was used to monitor the phase structure of many-flavor Wilson fermions at the strong coupling limit $(\beta=0)$ [55], and to study the chiral phase transition at finite temperature [56]. However, as long as we know, the properties of $\langle\bar{\psi} \psi\rangle_{\text {subt }}$ have not been investigated thus far. We will examine them in Sec. III before we use $\langle\bar{\psi} \psi\rangle_{\text {subt }}$ to study the occurence of chiral symmetry breaking in $N_{F}=6$ theory.

\section{E. finite size effect}

The simulation must be done for the system put in a finite box. All the quantities measured in the box thus receive more or less the effect due to this limitation. As is done in the statistical mechanics, as long as the size of the effect is small enough that it can be treated as a correction ${ }^{1}$, the information on the dependence of the quantities upon the system size and the boundary condition may help to extract the long distance dynamics of the system.

In this work, the finite size effect will actually play a crucial role to investigate the dynamical features of the target gauge theory. The purpose of this subsection is to provide the materials that form the basis of the forthcoming analysis in regard to this point; the summary of the known facts on the finite size effects in the system with spontaneous breakdown of chiral symmetry, simply abbreviated as $\chi$-theory, and the observation on those in the IR-conformal system.

1 We recall that the inverse of the lattice size, $1 / l=a / L$, is one of relevant perturbations. 
We consider first the pseudoscalar meson $P$ and the finite size effect on its mass $M_{P}$. If the linear size $L$ of the spatial volume becomes comparable to the Compton wavelength of $P$, the finite size correction $\Delta M_{P}(L)=M_{P}(L)-M_{P}$ due to the elastic scattering of $P$ is known to increase the mass, $\Delta M_{P}^{\mathrm{sc}}(L)>0$ [47, 48]. For instance, in $\mathrm{SU}(3)_{\mathrm{C}}$ gauge theory with two or three light quarks, where low-energy dynamics can be approximately described by Nambu-Goldstone bosons $\pi$, the $\pi \pi$ scattering effect dominates over the finite size effect on $M_{\pi}$ and thus increases it.

One is often inclined to identify the Compton wavelength $\sim 1 / M(L)$ with the spatial correlation length $\xi$. As $\xi$ cannot exceed the system size $L$, the mass is then bounded from below in proportion to $\sim 1 / L$, suggesting that the finite size effect increases $M(L)$.

However, the analysis in the framework of the quantum field theory implies that this is not always the case. As illustrated in Ref. [47], in pure Yang-Mills theory, the finite size effect acts on the mass $m_{G}$ of the glueball $G$ in the $0^{++}$-channel in such a way that it decreases $m_{G}(L)$, i.e. $\Delta M_{G}^{\text {tri }}(L)<0$, through the non-trivial trilinear coupling among glueballs with a dimensionful coefficient.

Now, let us suppose that the dynamics of the theory admits such a light $0^{++}$state $\sigma$ that is active in the low-energy effective theory for $P$. In case $\sigma$ is lighter than $P$, we can apply the result in Ref. [49], which generalizes Lüscher's analysis to the system of two species. The finite size effect decreases the mass of $P ; \Delta M_{P}(L) \simeq M_{P}^{\operatorname{tri}}(L)<0$, provided that it is dominated by the $\sigma P P$ interaction.

An example of negative $\Delta M_{P}(L)$ seems to be realized in the $\mathrm{SU}(2)_{\mathrm{C}}$ gauge theory with two adjoint fermions [27]. See Fig. 2 in Ref. [27]. Indeed, Fig. 11 in Ref. [27] shows that the $0^{++}$-glueball is lighter than the pseudoscalar meson $P$ in that theory.

Those results illustrate that the finite size effect reflects the dynamical features of the system and thus provides us a device to investigate them. In this paper, we would like to point out that the decay constant $f_{P}$ of the pseudoscalar meson could be also interesting from such a viewpoint.

We first recall that the chiral condensate $\langle\bar{\psi} \psi\rangle$ is an order parameter of the chiral symmetry. It thus inevitably vanishes in the chiral limit at finite volume. The situation is schematically represented as in Fig. 1 for the $\chi$-theory. Contrastingly, the decay constant $f_{P}$ is not the order parameter of the chiral symmetry in QCD or the gauge theory with fermionic matters in general. At finite volume, the linear size $L$ of the system provides the 


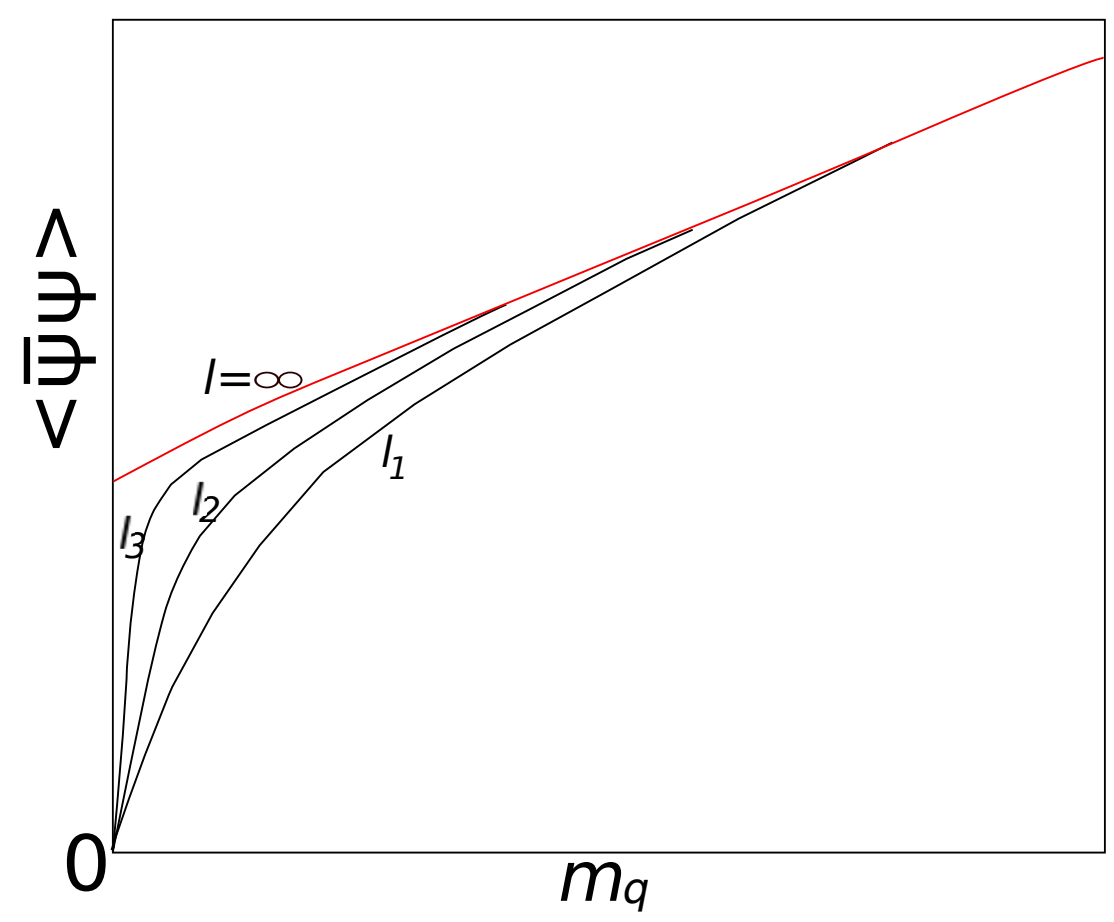

FIG. 1: $\langle\bar{\psi} \psi\rangle$ versus the quark mass at finite volume in the $\chi$-theory. Here, the linear sizes $l_{1}, l_{2}, l_{3}$ of three spatial volumes are ordered such that $l_{3}>l_{2}>l_{1}$. The condensate in the limit of the vanishing quark mass becomes nonzero only after the thermodynamic limit is taken first.

infrared cut off $\sim 1 / L$, and there is no symmetry reason that its chiral limit should vanish. In the $\chi$-theory, the chiral perturbation can be applied to the $p$-regime, $M_{\pi} \gg 1 / L$, and it is found that $f_{P}\left(m_{q}\right)$ at quark mass $m_{q}$ is reduced by the finiteness of system size for any $N_{F}$ as long as chiral symmetry breaking occurs and the parameters are in the $p$-regime [46, 57]. Also in the $\epsilon$-regime, $M_{\pi} \ll 1 / L$, the finiteness of system size acts to decrease $f_{P}\left(m_{q}\right)$, leaving a non-vanishing constant at $m_{q}=0$ even at finite volume [58]. Figure 2 shows the schematic dependence of $f_{P}\left(m_{q}\right)$ on quark mass $m_{q}$ and the linear size $L$ of the volume in the $\chi$-theory.

Now, we question how the finiteness of the system size affects to $f_{P}\left(m_{q}\right)$ in the IRconformal theory. The analysis in the mass-deformed conformal gauge theory together with the hyperscaling hypothesis [27, 28] shows that a scaling variable in the finite volume method is given by the combination $L m_{q}^{1 /\left(1+\gamma_{\star}\right)}$, where $\gamma_{\star}$ is the mass anomalous dimension at the infrared fixed point. However, it does not predict the form of the scaling function. In particular, it is uncertain whether the finite size effect tends to increase or decrease $f_{P}\left(m_{q}\right)$. Such a qualitative dependence of $f_{P}\left(m_{q}\right)$ on $L$ will reflect the dynamics of the system at 


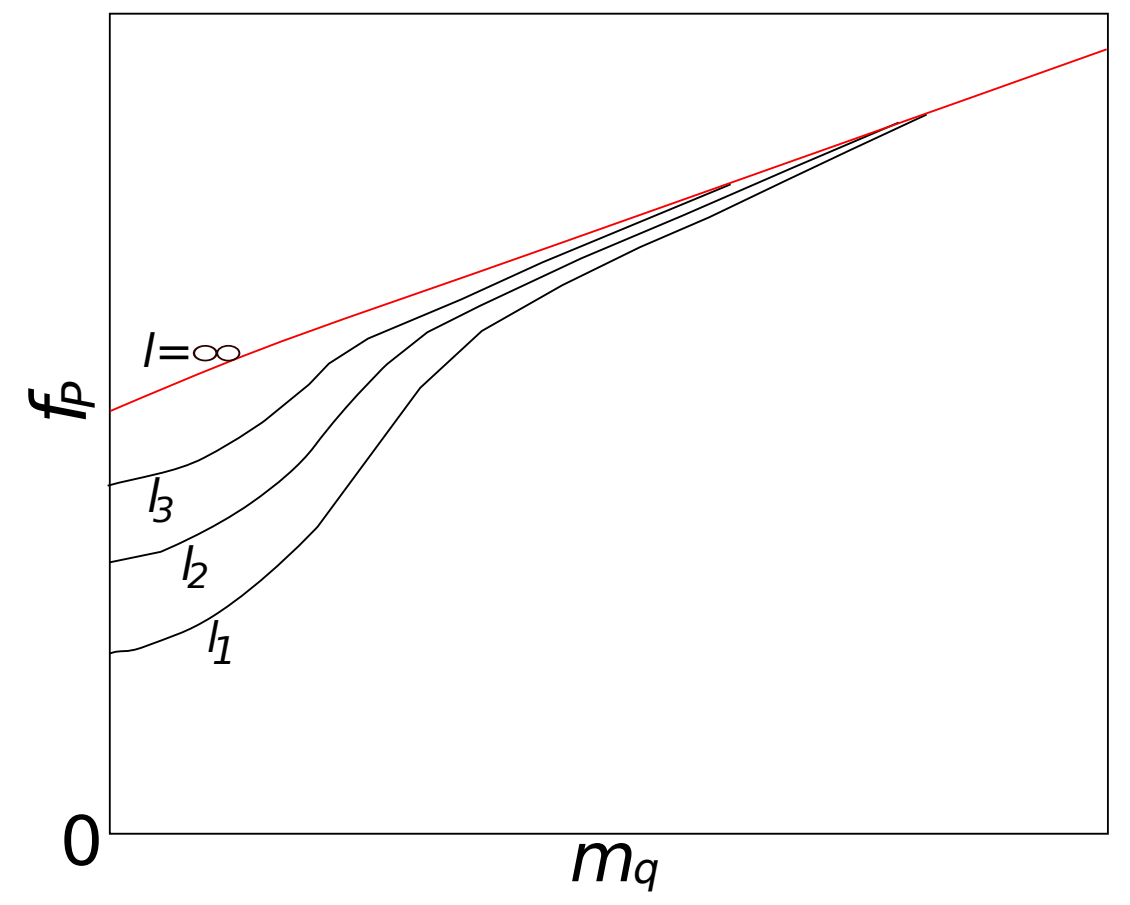

FIG. 2: The decay constant $f_{P}\left(m_{q}\right)$ versus the quark mass $m_{q}$ at finite volume in the $\chi$-theory.

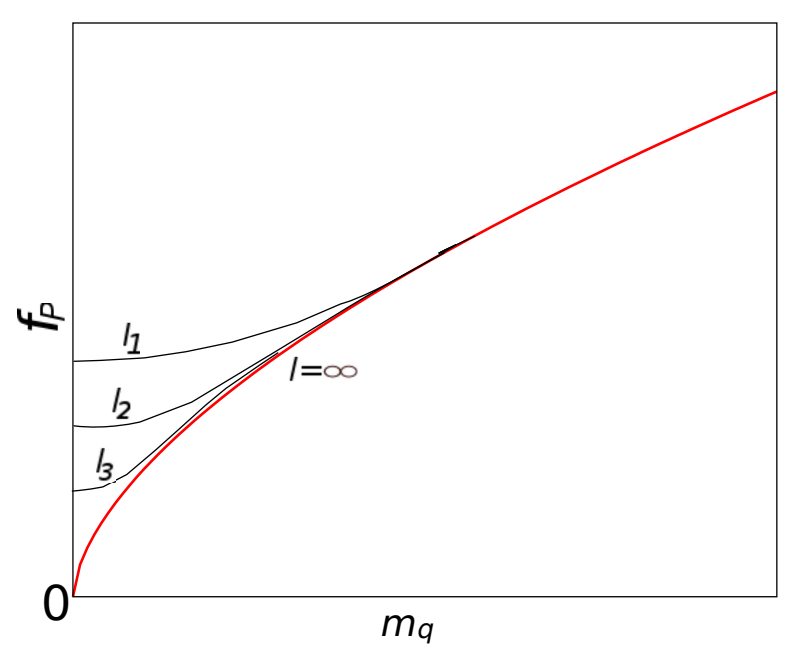

FIG. 3: Example 1 of possibility for finite size effect on the decay constant $f_{P}\left(m_{q}\right)$ versus the quark mass $m_{q}$ in the IR-conformal theory. The linear sizes $l_{1}, l_{2}, l_{3}$ of three spatial volumes are ordered such that $l_{3}>l_{2}>l_{1}$.

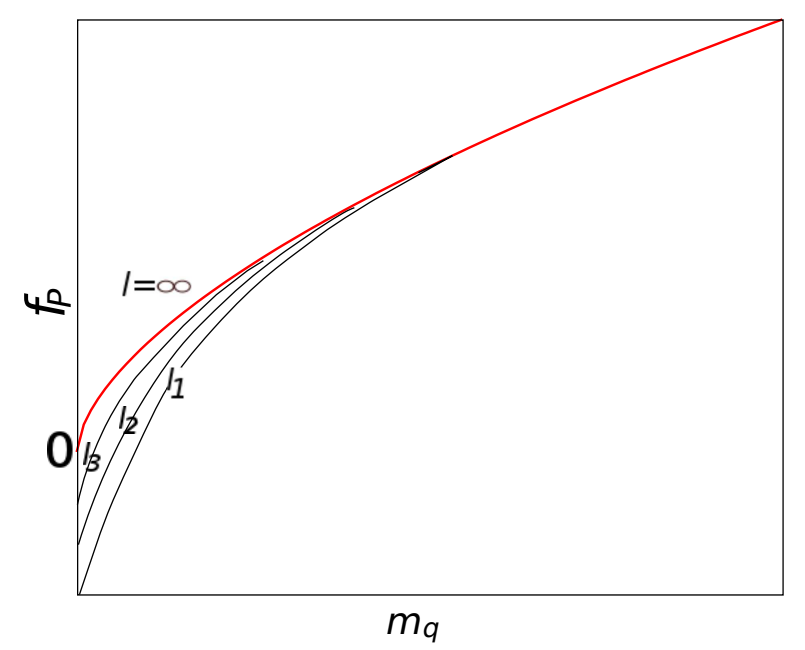

FIG. 4: Example 2 of possibility for finite size effect on the decay constant $f_{P}\left(m_{q}\right)$ versus the quark mass $m_{q}$ in the IR-conformal theory, in which $f_{P}$ is reduced by the finite size effect and approaches to a negative value in the chiral limit. 


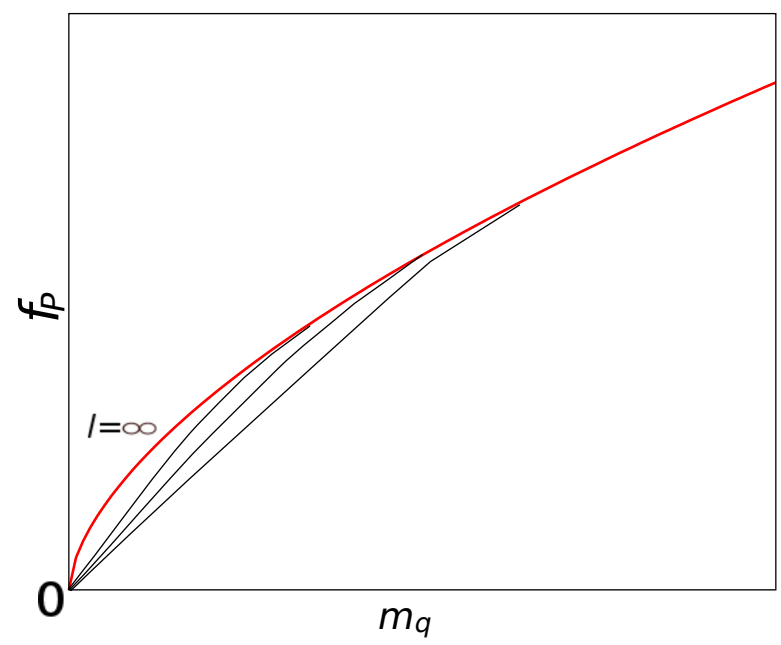

FIG. 5: Example 3 of possibility for finite size effect on the decay constant $f_{P}\left(m_{q}\right)$ versus the quark mass $m_{q}$ in the IR-conformal theory, in which $f_{P}$ becomes zero in the chiral limit.

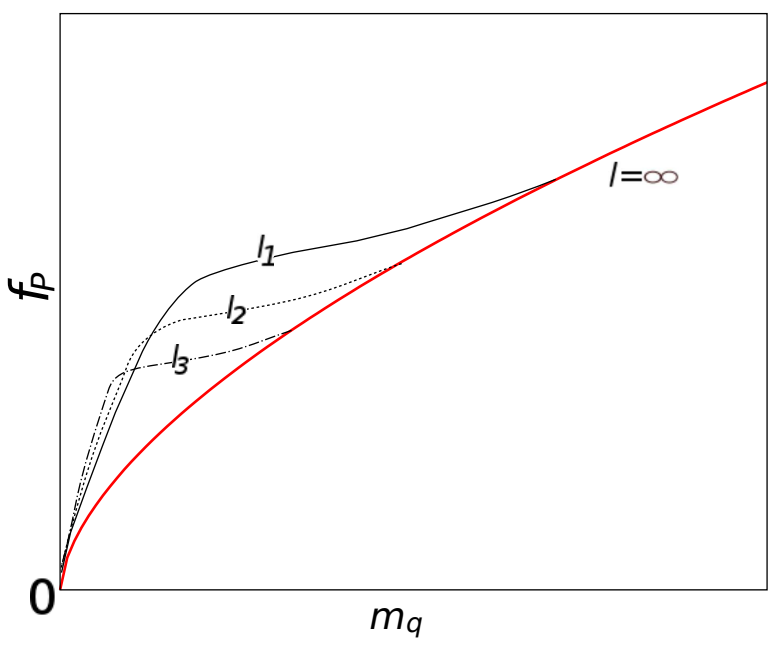

FIG. 6: Example 4 of the possibility for finite size effect on the decay constant $f_{P}\left(m_{q}\right)$ versus the quark mass $m_{q}$ in the IR-conformal theory, in which $f_{P}$ becomes zero in the chiral limit.

long distance. Here, we try to speculate various possibilities for the finite size effect on $f_{P}\left(m_{q}\right)$ in the theory without chiral symmetry breaking, in order to compare the measured data with them at the stage of the analysis. The hyperscaling hypothesis predicts that the universal part of the decay constant is given by the term $\propto\left(m_{q}\right)^{\alpha_{\star}}$ with $\alpha_{\star}=1 /\left(1+\gamma_{\star}\right)$ in the IR-conformal theory at infinite volume [27, 28], which is expected to dominate $f_{P}\left(m_{q}\right)$ if $\gamma_{\star}>0$. It is plausible that the finite size effect will show up at smaller $m_{q}$ at larger volume. With these "boundary conditions", we list four candidates of finite size effects in Figs. 3, 4, 5 and 6 .

In the example 1 (2) in Fig. 3 (44), the finite size effect is presumed to increase (decrease) $f_{P}$ with non-vanishing intercept in the chiral limit. Note that the example 1 can be clearly distinguished from the behavior in the $\chi$-theory as shown in Fig. 2 . In $\mathrm{SU}(2)_{\mathrm{C}}$ gauge theory with two adjoint Dirac fermions, the finite size effect appears to act on $f_{P}$ as in this example 1 , although it is not obvious that the intercept at $m_{q} \rightarrow 0$ is non-vanishing. (See Fig. 5 in Ref. [27].)

The case of the example 2 can be distinguished from the $\chi$-theory, because $f_{P}\left(m_{q}\right)$ is then expected to approach to zero at a certain non-zero $m_{q}$ in the finite volume theory. However, from Eq. (9) we can see that $A_{P P}$ vanishes at some quark mass in the example 2, implying 
the disappearance of contribution of the ground state in the $P P$ contribution. We are thus inclined to consider example 3 with vanishing intercept in the chiral limit as in Fig. 5, where the curves are further far away from the curve at $l=\infty$ for smaller volumes. If this case is realized, the finite size effect on $f_{P}$ may not help to determine whether the system is a X-theory or not. The example 4 in Fig [ 6 illustrates the case in which the decay constant $f_{P}$ is enhanced by the finite size effect but approaches to zero in the chiral limit.

\section{SU(2) $\mathrm{C}$ GAUGE THEORY WITH TWO-FLAVORS}

TABLE I: PCAC mass, chiral condensate, decay constant in two-flavor theory on $L / a=16$ and $\beta=2.0$ lattices.

\begin{tabular}{ccccc}
\hline \hline$\kappa$ & $2 a m_{\mathrm{PCAC}}$ & $a^{3}\langle\bar{\psi} \psi\rangle_{\text {subt }}$ & $a f_{P}$ \\
\hline $0.1540+0.6657(13)$ & $0.8861(18)$ & $0.2566(38)$ \\
$0.1580+0.4271(10)$ & $0.6346(14)$ & $0.2158(30)$ \\
$0.1620+0.1800(21)$ & $0.3055(37)$ & $0.1583(73)$ \\
$0.1625+0.1505(10)$ & $0.2624(18)$ & $0.1474(28)$ \\
$0.1630+0.1183(12)$ & $0.2116(21)$ & $0.1344(30)$ \\
\hline \hline
\end{tabular}

TABLE II: PCAC mass, chiral condensate, decay constant in two-flavor theory on $L / a=24$ and $\beta=2.0$ lattices.

\begin{tabular}{ccccc}
\hline \hline$\kappa$ & $2 a m_{\mathrm{PCAC}}$ & $a^{3}\langle\bar{\psi} \psi\rangle_{\mathrm{subt}}$ & $a f_{P}$ \\
\hline 0.1540 & $+0.6708(17)$ & $0.8935(23)$ & $0.2607(23)$ \\
$0.1620+0.18347(97)$ & $0.3113(19)$ & $0.1648(27)$ \\
$0.1625+0.15266(93)$ & $0.2656(21)$ & $0.1513(14)$ \\
$0.1630+0.11949(68)$ & $0.2140(13)$ & $0.1379(16)$ \\
$0.1637+0.06946(67)$ & $0.1317(13)$ & $0.1135(14)$ \\
\hline \hline
\end{tabular}

In this section, we observe the qualitative features of the finite size effects on the various hadronic quantities simulated for the $\chi$-theory at very weak gauge coupling. The simulation is performed in the $\mathrm{SU}(2)_{\mathrm{C}}$ gauge theory with two Dirac fermions in the fundamental representation, referred to as the two-flavor or $N_{F}=2$ theory. The data to be obtained for our 
TABLE III: Meson masses in two-flavor theory on $L / a=16$ and $\beta=2.0$ lattices.

\begin{tabular}{ccccc}
\hline \hline$\kappa$ & $a M_{P}$ & $a M_{V}$ & $a M_{S}$ & $a M_{A}$ \\
\hline 0.1540 & $1.1797(17)$ & $1.2446(28)$ & $2.083(82)$ & $2.048(44)$ \\
0.1580 & $0.9637(16)$ & $1.0525(29)$ & $1.856(81)$ & $1.833(53)$ \\
0.1620 & $0.6321(62)$ & $0.770(16)$ & $1.44(28)$ & $1.42(13)$ \\
0.1625 & $0.5797(32)$ & $0.7147(42)$ & $1.10(22)$ & $1.25(13)$ \\
0.1630 & $0.5155(33)$ & $0.6647(67)$ & $1.12(22)$ & $0.96(12)$ \\
\hline \hline
\end{tabular}

TABLE IV: Meson masses in two-flavor theory on $L / a=24$ and $\beta=2.0$ lattices.

\begin{tabular}{ccccc}
\hline \hline$\kappa$ & $a M_{P}$ & $a M_{V}$ & $a M_{S}$ & $a M_{A}$ \\
\hline 0.1540 & $1.1841(26)$ & $1.2452(29)$ & $2.20(14)$ & $2.091(82)$ \\
0.1620 & $0.6413(18)$ & $0.7647(39)$ & $1.53(11)$ & $1.41(20)$ \\
0.1625 & $0.5792(31)$ & $0.7082(63)$ & $1.132(93)$ & $1.329(70)$ \\
0.1630 & $0.5176(22)$ & $0.6543(37)$ & $1.08(18)$ & $1.060(93)$ \\
0.1637 & $0.3930(25)$ & $0.5519(48)$ & $1.192(74)$ & $1.137(42)$ \\
\hline
\end{tabular}

target system, the six-flavor theory, will be confronted with the knowledge and experiences obtained in this section. We also examine the utility of the subtracted chiral condensate (11) by looking at the data in the two-flavor theory.

To highlight the finite size effects, the simulation is first performed in the two-flavor theory at $\beta \equiv 4 / g_{0}^{2}=2.0$ with lattice sizes, $16^{3} \times 64$ and $24^{3} \times 64$. Tables 【 and II list the simulation parameters, and the results for PCAC masses, subtracted chiral condensate $\langle\bar{\psi} \psi\rangle_{\text {subt }}$ and decay constant $f_{P}$, for $L / a=16$ and $L / a=24$, respectively. The gauge configurations are stored once every 10 trajectories. The number of configurations used in the measurement is $100 \sim 300$, depending on the parameters. Statistical errors are estimated by the single elimination jack-knife method throughout this work. The auto-correlation among the configurations is checked by binning data and varying the bin size.

The masses of lightest mesons in the various flavor-nonsinglet channels are shown in Tabs. III and IV, Note that the effective mass plots in the scalar and axial-vector channels 
exhibit large fluctuation at $t$ greater than $8 \sim 10^{2}$. Thus, the masses of these channels are obtained by fit at smaller $t$ and the limited range if required.

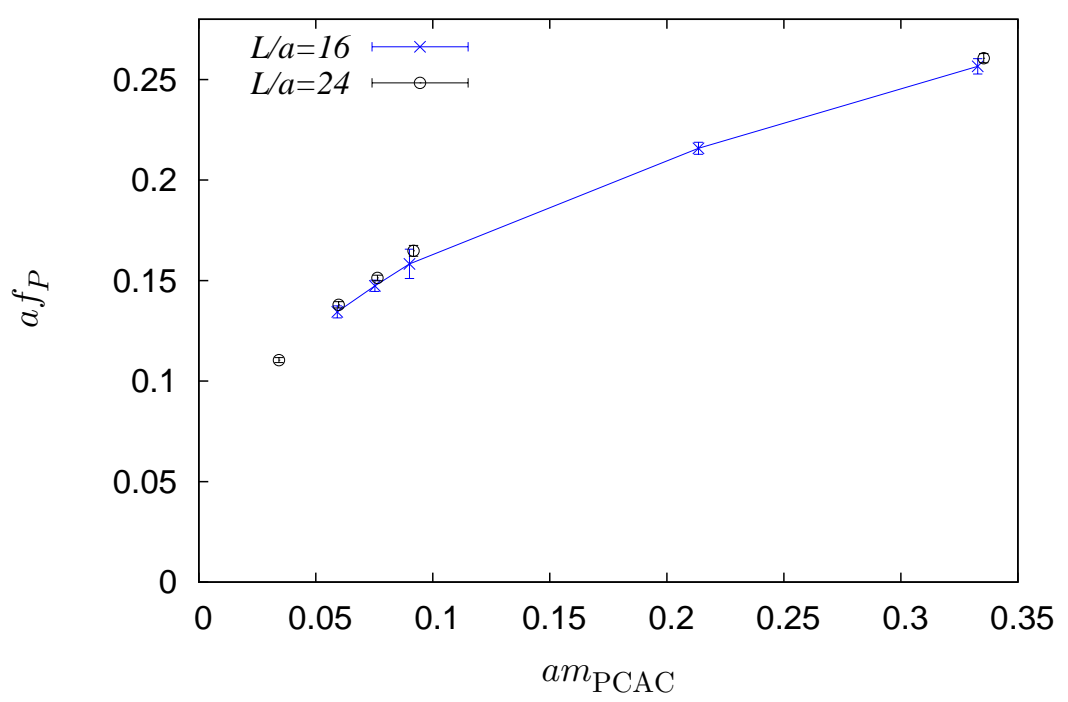

FIG. 7: Decay constant $f_{P}$ in two-flavor theory at $\beta=2.0$ and two different volumes, $16^{3}$ (blue cross dots) and $24^{3}$ (black circle dots). The line is written just to guide eyes to the plots of $L / a=16$.

Figures 7 and 8 show the quark mass dependence of decay constant $f_{P}$ and subtracted chiral condensate, respectively. At present, no explicit analysis for the finite size effect on $f_{P}$ is available according to the chiral perturbation theory for the breaking pattern $\mathrm{SU}\left(2 N_{F}\right) \rightarrow \mathrm{Sp}\left(2 N_{F}\right)$ anticipated in the $\mathrm{SU}(2)_{\mathrm{C}}$ gauge theory. In Fig. 7 the finite size effect is small but seems to tend to decrease $f_{P}{ }^{3}$. This is compatible with the schematic behavior in Fig. 2 predicted from the chiral perturbation theory for $\mathrm{SU}\left(N_{F}\right)_{\mathrm{L}} \times \mathrm{SU}\left(N_{F}\right)_{\mathrm{R}} \rightarrow \mathrm{SU}\left(N_{F}\right)_{\mathrm{V}}$ in the $\mathrm{SU}(3)_{\mathrm{C}}$ gauge theory with chiral symmetry breaking.

As mentioned in Sec. IID, we will use the subtracted chiral condensate $\langle\bar{\psi} \psi\rangle_{\text {subt }}$ in Eq. (11) as one of the quantities to examine the occurrence of spontaneous chiral symmetry

2 Such large fluctuation does not appear for small quark both at weak coupling in $N_{F}=2$ theory and at $\beta=2.0$ in $N_{F}=6$ theory.

3 The tendency of decrease of $f_{P}$ can be more clearly observed in Fig. 9 for $B$ which is proportional to $1 / f_{P}^{2}$, together with the fact that the subtracted chiral condensate $\langle\bar{\psi} \psi\rangle_{\text {subt }}$ does not suffer visible finite size effect. 


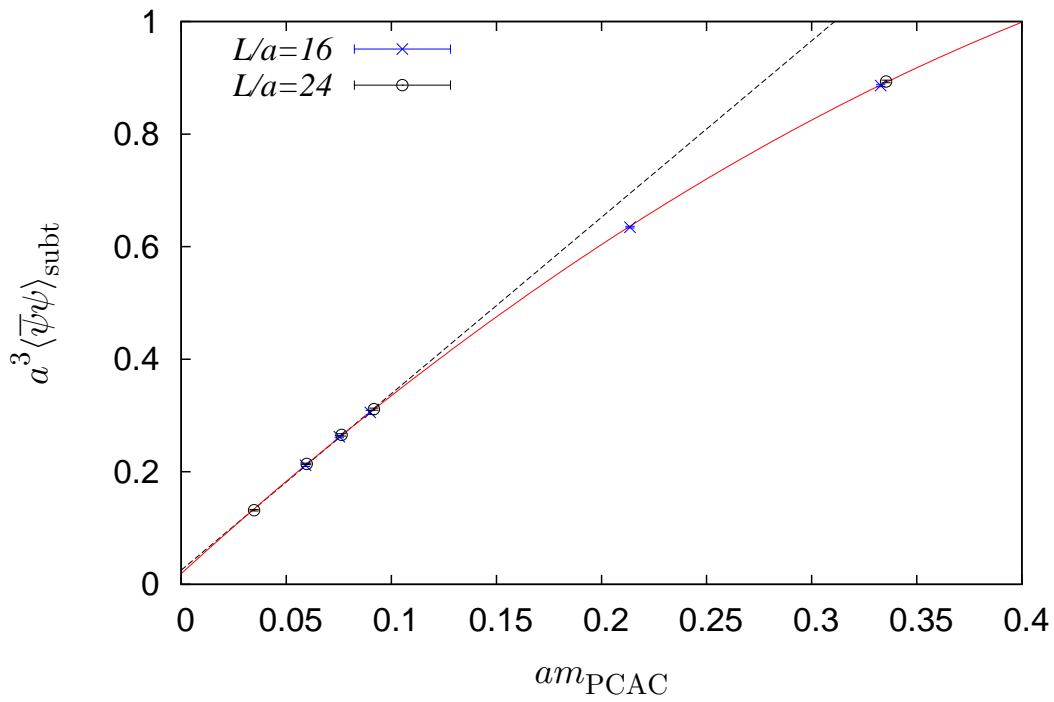

FIG. 8: Subtracted chiral condensate in two-flavor theory at $\beta=2.0$ and two different volumes, $16^{3}$ (blue cross dots) and $24^{3}$ (black circle dots). No detectable finite size effect is observed, and the linear fit (dotted line) to the data of quark masses $a m_{\mathrm{PCAC}}<0.1$ and the quadratic fit to all data are performed. Only the fit curves with the central values for the coefficients are drawn.

breaking in the foregoing analysis. Here, we discuss the utility of $\langle\bar{\psi} \psi\rangle_{\text {subt }}$.

The subtracted chiral condensate $\langle\bar{\psi} \psi\rangle_{\text {subt }}$ is dominated by the contribution proportional to $m_{\mathrm{PCAC}}$ and its chiral limit seems to be well below $O(1)$ in the lattice unit. The result for $\langle\bar{\psi} \psi\rangle_{\text {subt }}$ at $\beta=2.0$ in the two-flavor theory is shown in Fig. 8, which implies that it is actually the case. This is the first indication of the utility of $\langle\bar{\psi} \psi\rangle_{\text {subt }}$ as the counterpart of the chiral condensate in the continuum. Contrastingly, the chiral limit of $\langle\bar{\psi} \psi\rangle$ directly calculated as in Eq. (10) is $O(1)$ in the lattice unit, reflecting the dominance of cubic UV divergence.

From Eq. (11), the subtracted chiral condensate is proportional to $m_{\mathrm{PCAC}}$. Therefore, in order for $\langle\bar{\psi} \psi\rangle_{\text {subt }}$ to produce a finite and non-vanishing VEV in the thermodynamic limit, the four-volume sum of pseudoscalar correlator must diverge linearly with respect to $m_{\mathrm{PCAC}}$. The four-volume sum is regular with respect to the quark mass at finite volume, and a singularity is possibly developed only in the infinite volume limit, compatible with Fig. 1. Practically, what we can do at best is to examine if the data are not incompatible with the non-vanishing VEV in the chiral limit as follows. First, no finite size effect can be seen in 
the existing data shown in Fig. 8. It is thus plausible to consider that they approximate the mass dependence in the infinite volume very well (Unexpectedly, the size is too small to take the thermodynamic limit.). Meanwhile, Fig. 8 indicates that the shape of the mass dependence of the subtracted chiral condensate is convex upward. The linear extrapolation to such data will thus tend to overestimate the chiral limit. Despite this fact, we first try to fit a linear function

$f_{2}\left(x=a m_{\mathrm{PCAC}}\right)=a_{0}+a_{1} x$

to the data of $a m_{\mathrm{PCAC}}<0.1$, and get the result

$a_{0}=0.0250(28), \quad a_{1}=3.136(38)$.

We also fit a quadratic function

$f_{3}\left(x=a m_{\mathrm{PCAC}}\right)=b_{0}+b_{1} x+b_{2} x^{2}$,

to all the data, and find

$b_{0}=0.0190(19), \quad b_{1}=3.397(29), \quad b_{2}=-2.368(74)$.

Both are not incompatible with the non-vanishing VEV in the thermodynamic limit.

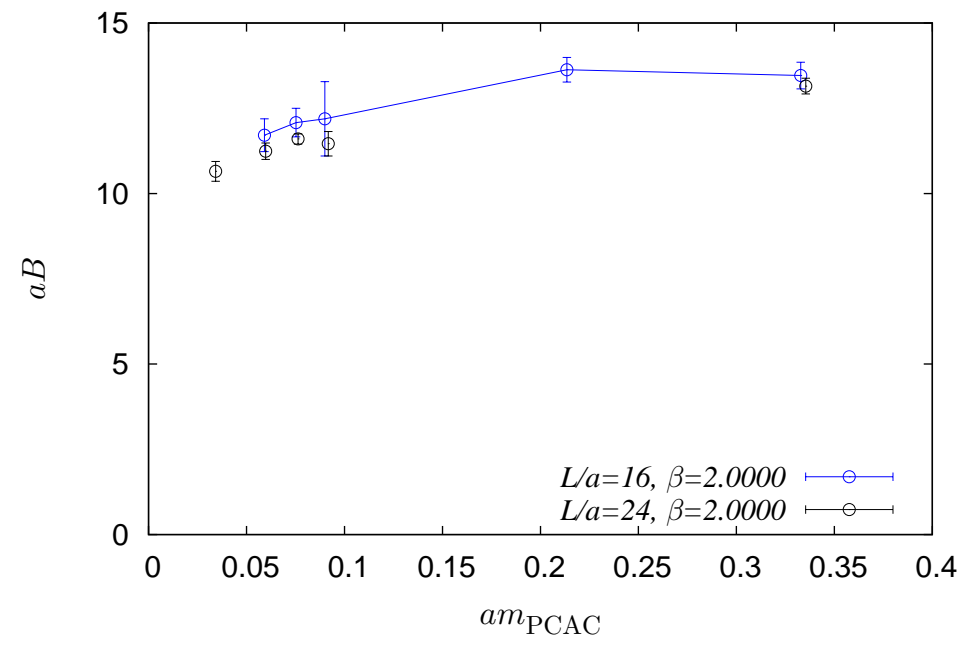

FIG. 9: $B$ defined by Eq. (16) in two-flavor theory at $\beta=2.0$.

For a given quark mass and volume, we define $a B$ by

$a B \equiv a \frac{\langle\bar{\psi} \psi\rangle_{\text {subt }}}{f_{P}^{2}}$. 
The parameter in the chiral perturbation theory, $B_{0}$ [59], is given by the same equation but with the renormalized condensate and decay constant in the chiral limit. $B_{0}$ will thus be obtained by multiplicative renormalization of $\left.B\right|_{m_{\mathrm{PCAC} \rightarrow 0}}$. As our main purpose is to explore qualitative dynamics at long distance, e.g. finiteness and non-vanishment in the chiral limit, use of $B$ will suffice. Figure 9 shows $B$ calculated by Eq. (16) with use of the subtracted chiral condensate $\langle\bar{\psi} \psi\rangle_{\text {subt }}$ at $\beta=2.0$ in the two-flavor theory. It indicates that $B$ is finite and non-vanishing in the chiral limit. Those features give another support for the utility of the subtracted chiral condensate.

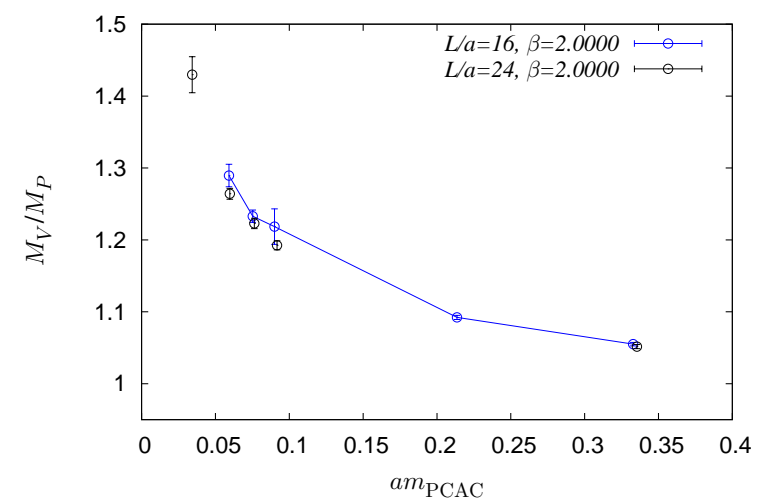

FIG. 10: $M_{V} / M_{P}$ in two-flavor theory at $\beta=$ 2.0 .

Figures 10 and 11 show the ratios $M_{V} / M_{P}$ and $f_{P} / M_{P}$, respectively, both of which increase for smaller quark mass as expected in the $\not$-theory such as the two-flavor $\mathrm{SU}(2)_{\mathrm{C}}$ gauge theory.

TABLE V: PCAC mass, chiral condensate, decay constant in two-flavor theory on $L / a=16$ and $\beta=4.0$ lattices.

\begin{tabular}{cccc}
\hline \hline$\kappa$ & $2 a m_{\mathrm{PCAC}}$ & $a^{3}\langle\bar{\psi} \psi\rangle_{\text {subt }}$ & $a f_{P}$ \\
\hline $0.1280+0.39760(38)$ & $0.29195(27)$ & $0.04980(75)$ \\
$0.1320+0.17976(19)$ & $0.14372(16)$ & $0.03591(38)$ \\
$0.1340+0.06456(32)$ & $0.05377(27)$ & $0.01551(16)$ \\
\hline \hline
\end{tabular}

Next, the simulation is performed at relatively weak coupling, $\beta=4.0$, and sizes of 
TABLE VI: PCAC mass, chiral condensate, decay constant in two-flavor theory on $L / a=24$ and $\beta=4.0$ lattices.

\begin{tabular}{cccc}
\hline \hline$\kappa$ & $2 a m_{\mathrm{PCAC}}$ & $a^{3}\langle\bar{\psi} \psi\rangle_{\text {subt }}$ & $a f_{P}$ \\
\hline $0.1280+0.3926(12)$ & $0.28830(9)$ & - \\
$0.1320+0.16795(10)$ & $0.13451(8)$ & $0.03457(18)$ \\
$0.1340+0.05630(11)$ & $0.047016(9)$ & $0.01581(16)$ \\
\hline \hline
\end{tabular}

TABLE VII: Meson masses in two-flavor theory on $L / a=16$ and $\beta=4.0$ lattices. The column is left in blank unless the effective mass plots obtained from the point-source exhibit plateau.

\begin{tabular}{ccccc}
\hline \hline$\kappa$ & $a M_{P}$ & $a M_{V}$ & $a M_{S}$ & $a M_{A}$ \\
\hline 0.1280 & $0.5547(35)$ & $0.5582(34)$ & $0.5710(43)$ & $0.5745(44)$ \\
0.1320 & $0.5970(33)$ & - & $0.6019(32)$ & - \\
0.1340 & $0.5987(33)$ & $0.6096(34)$ & $0.6003(34)$ & $0.6112(34)$ \\
\hline \hline
\end{tabular}

lattices, $16^{3} \times 64$ and $24^{3} \times 64$, in which the average plaquette, $\langle W\rangle$, where

$W \equiv \frac{1}{n_{U}} \sum_{n \in \Gamma_{4}} \sum_{\mu<\nu} \frac{1}{N_{C}} \operatorname{tr} U(n ; \mu, \nu)$,

with $n_{U}$ the total number of the summed plaquettes, is around 0.8. The simulation parameters are listed in Tabs. $\mathrm{V}$ and $\mathrm{VI}$.

Figure 12 shows $a^{3}\langle\bar{\psi} \psi\rangle_{\text {subt }}$ measured at $\beta=4.0$ together with the result for the fit to the data with $a m_{\mathrm{PCAC}} \leq 0.1$ as they show inappreciable finite volume correction. The value in the chiral limit is found to be

$$
\left.\lim _{m_{\mathrm{PCAC}} \rightarrow 0} a^{3}\langle\bar{\psi} \psi\rangle_{\mathrm{subt}}\right|_{\beta=4.0}=\left\{\begin{array}{l}
0.00310(18) \text { linear fit } \\
0.0016(14) \text { quadratic fit }
\end{array},\right.
$$

TABLE VIII: Meson masses in two-flavor theory on $L / a=24$ and $\beta=4.0$ lattices.

\begin{tabular}{ccccc}
\hline \hline$\kappa$ & $a M_{P}$ & $a M_{V}$ & $a M_{S}$ & $a M_{A}$ \\
\hline 0.1280 & - & - & - & - \\
0.1320 & $0.3085(37)$ & $0.3179(21)$ & $0.3231(31)$ & $0.3312(41)$ \\
0.1340 & $0.2717(22)$ & - & $0.2720(40)$ & - \\
\hline \hline
\end{tabular}




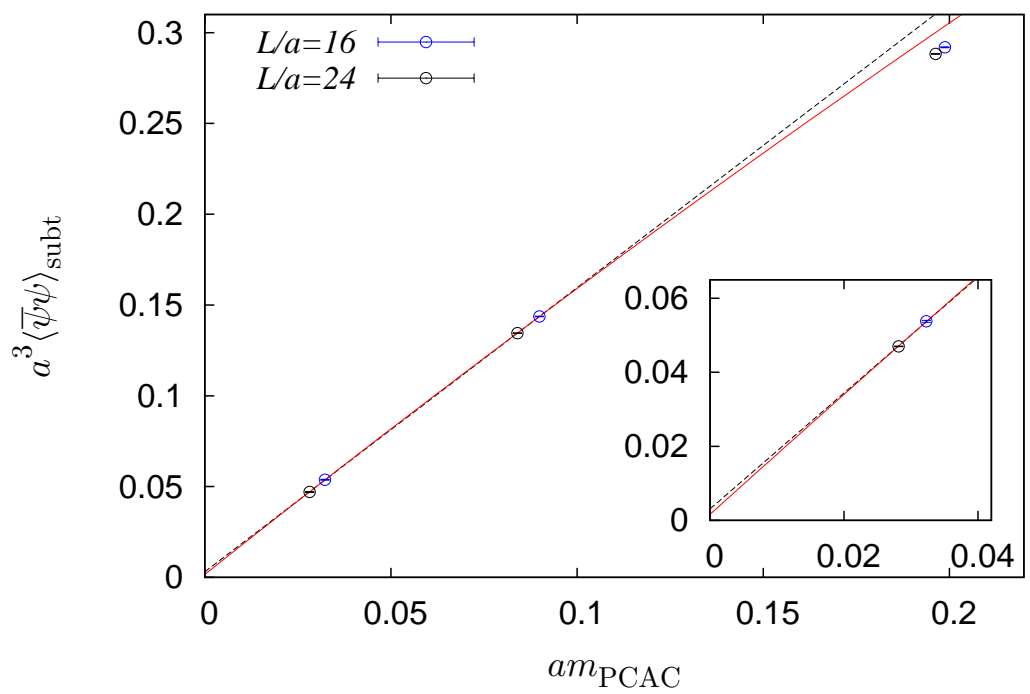

FIG. 12: Subtracted chiral condensate in two-flavor theory at $\beta=4.0$ in two different volumes, $16^{3}$ (blue cross dots) and $24^{3}$ (black circle dots). The linear fit (black dotted line) and the quadratic fit (red curve) to such data that satisfy $a m_{\mathrm{PCAC}} \leq 0.1$ are performed.

in this case.

Next, we turn our attention to $a f_{P}$ at $\beta=4$.0. Figure 13 indicates that $a f_{P}$ approaches to 0 in the limit $m_{\mathrm{PCAC}} \rightarrow 0$ at finite volume. To find the reason of this behavior, we plot the mass $M_{P}$ of the pseudoscalar meson for $\beta=4.0$ in Fig. 14. In contract to Fig. [15] at $\beta=2.0$, $M_{P}$ is bounded from below and does not seem to approach to 0 in the limit $m_{\mathrm{PCAC}} \rightarrow 0$. The simulated lattices with $L / a=16$ and $a m_{\mathrm{PCAC}}<0.1$ belong to the region where $M_{P}$ no longer decreases. Figure 14 also shows that the size of the lower bound depends on the volume. Hence, the lower bound originates from the finite size effect. Since $a f_{P}$ is calculated by Eq. (9), the chiral extrapolation of the data of $f_{P}$ at finite volume in the region where $M_{P}$ almost ceases to decrease eventually yields zero in the massless quark limit, even in the $\chi$-theory. In Figure 13, visible finite size effect is observed in $f_{P}$, and thus the chiral limit should be taken for a set of values that are obtained from those raw data in the infinite volume extrapolation. We recall that the result of the finite volume correction shown in Fig. 2 assumes $4 \pi F_{P} \cdot L \gg 1$ for $\left.F_{P} \equiv f_{P}\right|_{L / a \rightarrow \infty, m_{\mathrm{PCAC} \rightarrow 0}}$, which is presumably not satisfied for all of our lattices at $\beta=4.0$.

Since $f_{P}$ decreases while $M_{P}$ is bounded from below, the ratio $f_{P} / M_{P}$ at each lattice size 


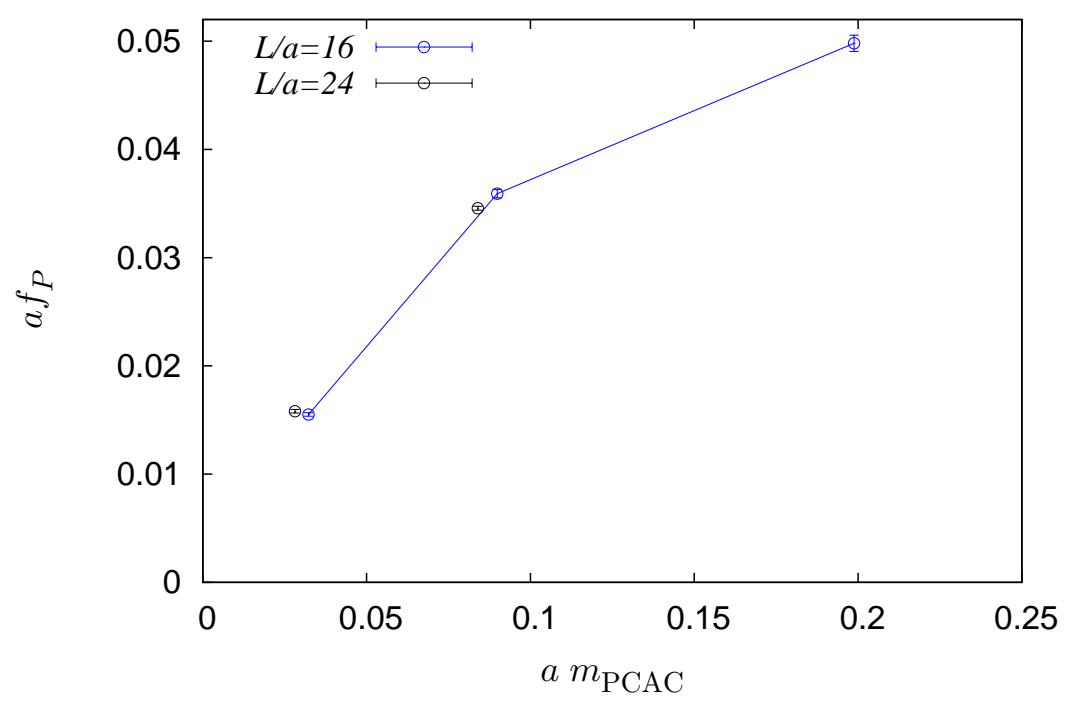

FIG. 13: Decay constant $f_{P}$ in two-flavor theory at $\beta=4.0$ in two different volumes, $16^{3}$ (blue cross dots) and $24^{3}$ (black circle dots).

also decreases to zero in the limit $m_{\mathrm{PCAC}} \rightarrow 0$, as in Fig. 16. We have seen in Fig. 12 that the chiral condensate possibly approaches to a non-vanishing value in the chiral limit. Since $f_{P}$ approaches to zero, $B$ defined by Eq. (16) will diverge in the chiral limit at finite volume. This explains the behavior observed in Fig. 17.

For future reference, we summarize our findings in the weak coupling simulation for the system with chiral symmetry breaking:

1. Meson masses stop to decrease in the chiral limit at finite volume, with the sizedependent lower bound. Pseudoscalar meson tends to form a pair with scalar meson after the lower bound is reached. (See Tabs. VII and VIII.)

2. The decay constant $f_{P}$ calculated by Eq. (9) approaches to zero in the chiral limit at finite volume.

3. $f_{P} / M_{P}$ approaches to zero in the chiral limit at finite volume even in the $\chi$-theory.

4. $B\left(m_{\mathrm{PCAC}}\right)$ defined by Eq. (16), which is seen to approach to a finite value at the modest coupling constant $\beta=2.0$, blows up in limit $m_{\mathrm{PCAC}} \rightarrow 0$ at finite volume. 


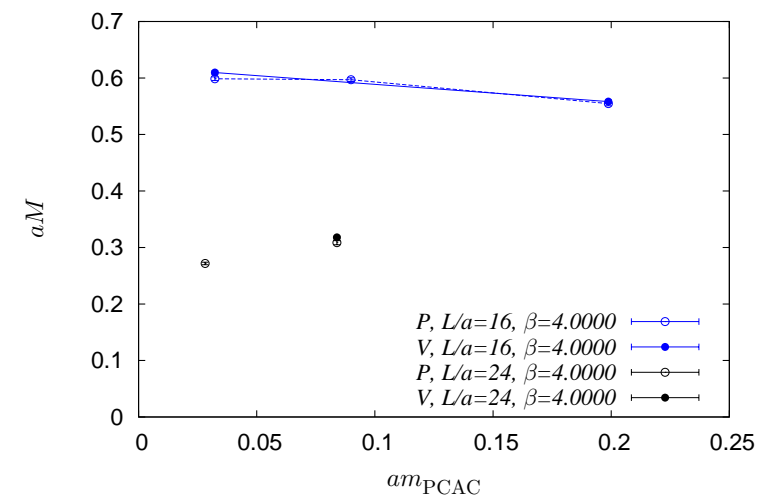

FIG. 14: $M_{P}$ (dashed line) and the lightest vector meson mass $M_{V}$ in two-flavor theory at $\beta=4.0$. The difference between $M_{V}$ and $M_{P}$ is negligibly small, and both of them are bounded from below at small quark masses.

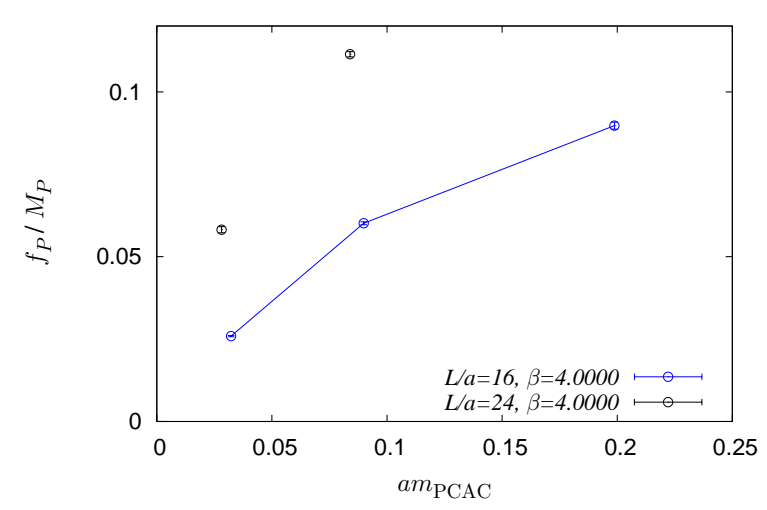

FIG. 16: $f_{P} / M_{P}$ in two-flavor theory at $\beta=$ 4.0.

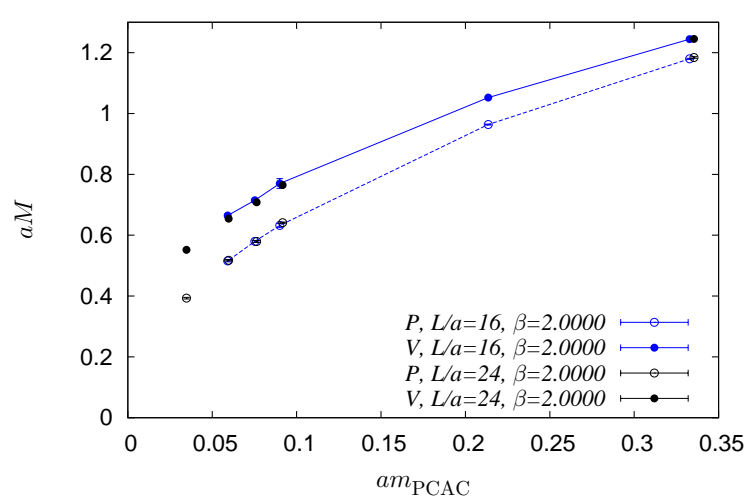

FIG. 15: $M_{P}$ (dashed line) and the lightest vector meson mass $M_{V}$ in two-flavor theory at $\beta=2.0$. 


\section{BRIEF LOOK AT PHASE STRUCTURE OF TWO-COLOR SIX-FLAVOR WILSON FERMIONS}

This work is intended to provide the information complementary to that found by the study of the running coupling constant in the Schrödinger functional scheme [44]. In the latter, the calculation is performed at the massless points $\kappa_{c}(\beta)$ for a number of $\beta$, and the continuum limit is taken explicitly. Rather, this work focuses on the dependence on quark masses of the various quantities, which requires the computation at various $\kappa$. As the first exploratory study, we perform the simulation at a fixed value of $\beta$, and forgive taking the explicit continuum limit. In this section, we examine the choice for its appropriate value.

As seen in Sec. IIA, no dimensionful parameter exists in the the lattice action. The lattice spacing $a$ and the physical size $L$ of the system is controlled mostly by the bare coupling constant $g_{0}^{2}=4 / \beta$. The lattice with geometry $l^{3} \times N_{T}$ represents smaller $a$ and physical size $L=l a$ at the weaker coupling in the asymptotically free gauge theory. As the number of flavors is increased, the effective coupling runs more slowly. Thus, to capture the strong coupling dynamics of the $\mathrm{SU}(2)_{\mathrm{C}}$ gauge theory with six flavors, the simulation with fine lattices requires very large size of lattice and vast statistics to sample gauge fields with non-trivial topology, which cannot be done with the available computational resources. In this work, we attempt to study the dynamics of the six-flavor theory by carrying out the simulation on coarse lattices. The results will be checked by making a comparison with the results found in the Schrödinger functional scheme in which the continuum limit is explicitly taken. We thus start with choosing an appropriate value of $\beta$ which does not belong to the strong coupling phase peculiar to the lattice action (2) with Wilson fermions. For that purpose, it is inevitable to study more or less the phase structure of the system with six Wilson fermions.

Contrary to the work [20], which surveys the phase structure of many flavor Wilson lattice gauge theories to explore the aspects of the systems, our aim is very restrictive here; we circumvent the parameters corresponding to the strong coupling phase which is separated from the weak coupling phase by the first-order bulk phase transition or the sharp crossover. Since such an unwanted strong coupling phase are attributed to the structure at short distance, we monitor the transition by the VEV of the averaged plaquettes, $\langle W\rangle$, where and the PCAC mass $a m_{\mathrm{PCAC}}$ defined in Sec. IIC, using the lattices of sizes, $8^{3} \times 32$ 


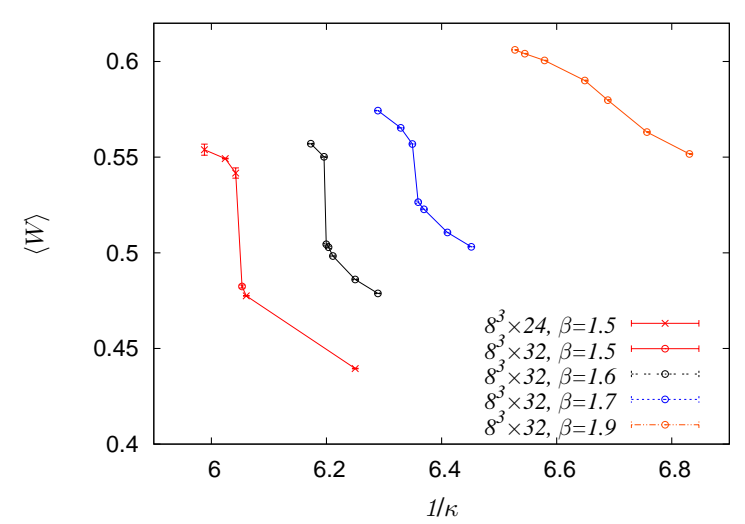

FIG. 18: $\langle W\rangle$ as a function of $1 / \kappa$. Lines are added just to guide eyes.

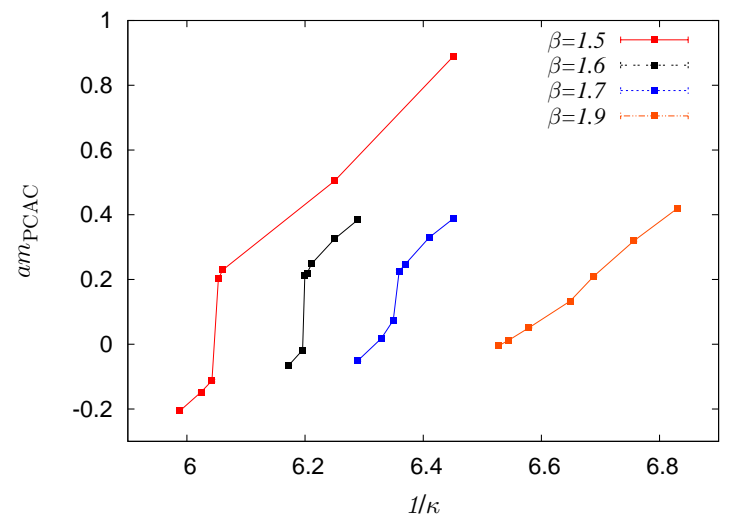

FIG. 19: PCAC mass $a m_{\mathrm{PCAC}}$ as a function of $1 / \kappa$. The results of $8^{3} \times 24$ and $8^{3} \times 32$ are plotted on the same footing for $m_{\mathrm{PCAC}}$ at $\beta=1.5$.

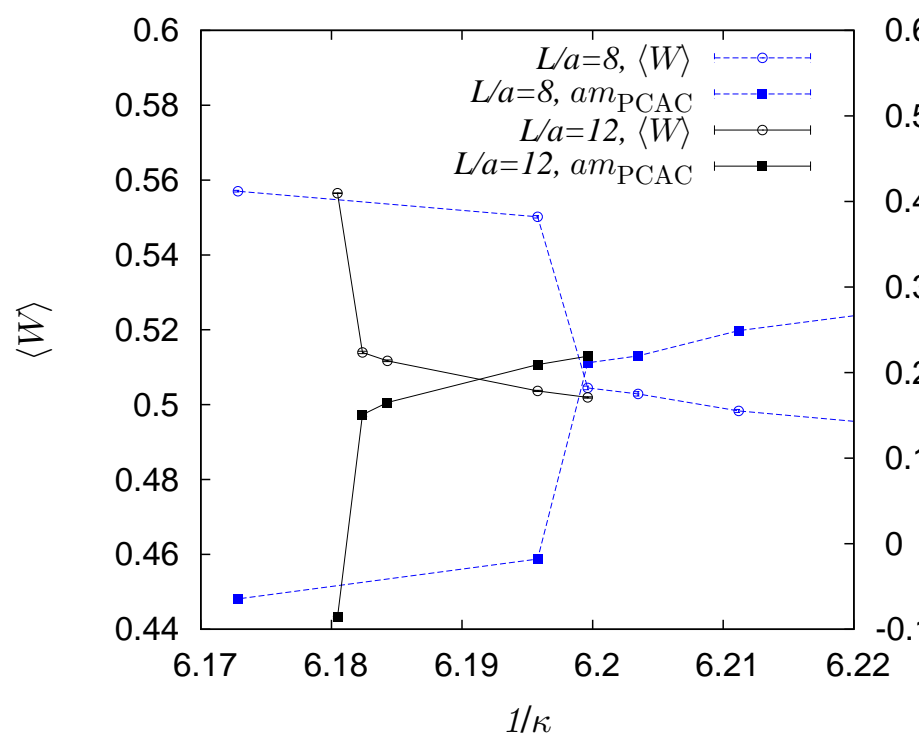

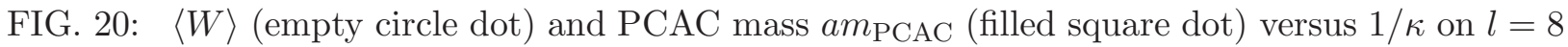
(dashed blue curve) and $l=12$ (black curve).

and $8^{3} \times 24$. The result is shown in Figs. 18 and 19. Discontinuous or abrupt change of $\langle W\rangle$ for the plaquette (17) and $a m_{\mathrm{PCAC}}$ at some $\kappa$ is observed for $\beta \lesssim 1.7$.

In order to examine the property of the transition, simulation is performed on larger lattices, $12^{3} \times 64$, at $\beta=1.6$, with a focus put on the vicinity of the transition point. Figure 20 shows that the values for the hopping parameter, $\kappa_{t}(L / a)$, at which the transition 
occurs are the same within $1 \%$ between two volumes, indicating that the transition is not related to any long distance physics. With these results, we choose $\beta=2.0$ as a simulation parameter. It will then turn out that $a m_{\mathrm{PCAC}}$ depends nearly linearly on the inverse of the hopping parameter, $1 / \kappa$, at $\beta=2.0$. (See Fig. 21.) 


\section{RESULTS}

In this section, we present the results of the lattice simulation carried out for the $\mathrm{SU}(2)_{\mathrm{C}}$ gauge theory with six-flavors. We give three signatures supporting that the six-flavor theory is not the one with chiral symmetry breaking.

TABLE IX: PCAC mass, chiral condensate, decay constant in six-flavor theory on $L / a=16$ and $\beta=2.0$ lattices. The latticee geometry is $16^{3} \times 32$ for $\kappa \leq 0.14300$, and $16^{3} \times 64$ for $\kappa \geq 0.14500$.

\begin{tabular}{cccc}
\hline \hline$\kappa$ & $2 a m_{\mathrm{PCAC}}$ & $a^{3}\langle\bar{\psi} \psi\rangle_{\mathrm{subt}}$ & $a f_{P}$ \\
\hline $0.13800+1.3073(69)$ & $1.1942(63)$ & $0.2268(68)$ \\
$0.14100+0.9848(64)$ & $0.9644(63)$ & $0.2015(65)$ \\
$0.14300+0.7686(30)$ & $0.7878(29)$ & $0.1859(46)$ \\
$0.14500+0.5423(10)$ & $0.5830(11)$ & $0.1424(14)$ \\
$0.14600+0.4335(10)$ & $0.4772(12)$ & $0.12697(78)$ \\
$0.14700+0.3197(10)$ & $0.3603(11)$ & $0.1024(17)$ \\
$0.14750+0.2663(8)$ & $0.3034(9)$ & $0.08803(14)$ \\
$0.14760+0.2572(8)$ & $0.2936(10)$ & $0.0892(13)$ \\
$0.14770+0.2470(8)$ & $0.2828(9)$ & $0.09006(67)$ \\
$0.14785+0.2285(10)$ & $0.2623(12)$ & $0.0858(19)$ \\
$0.14800+0.2133(10)$ & $0.2455(12)$ & $0.0799(15)$ \\
$0.14900+0.1102(5)$ & $0.12917(57)$ & $0.04425(74)$ \\
$0.14950+0.06524(54)$ & $0.07711(64)$ & $0.02673(32)$ \\
\hline \hline
\end{tabular}

The lattices used in this work are all homogeneous in the spatial directions. The number of the sites along each direction, $l=L / a$, is either $l=16,24$ or 32 in this work. As explained above, the inverse of the gauge coupling constant $\beta$ is set to 2.0 for all lattices. The hopping parameters are summarized in Tab. IX for $l=16$, Tab. X] for $l=24$, and Tab. XI for $l=32$, respectively. The gauge configurations are stored once every 10 trajectories for $L / a=16$ and 24, and the measurements are performed for $100 \sim 300$ configurations depending on parameters. For $L / a=32$, the configurations are stored once every 5 trajectories for $L / a=32$ owing to the upper limit on the time available to a single job. The measurements are carried out for more than 400 configurations. 
TABLE X: PCAC mass, chiral condensate, decay constant in six-flavor theory on $L / a=24$ and $\beta=2.0$ lattices. All lattices has the same geometry $24^{3} \times 48$.

\begin{tabular}{cccc}
\hline \hline$\kappa$ & $2 a m_{\mathrm{PCAC}}$ & $a^{3}\langle\bar{\psi} \psi\rangle_{\text {subt }}$ & $a f_{P}$ \\
\hline $0.14100+0.9833(21)$ & $0.9623(20)$ & $0.2054(34)$ \\
$0.14300+0.7629(16)$ & $0.7825(16)$ & $0.1816(27)$ \\
$0.14400+0.6541(21)$ & $0.6867(22)$ & $0.1626(18)$ \\
$0.14500+0.5429(17)$ & $0.5839(18)$ & $0.1491(21)$ \\
$0.14600+0.4345(17)$ & $0.4787(19)$ & $0.1258(14)$ \\
$0.14700+0.3229(9)$ & $0.3639(10)$ & $0.1012(7)$ \\
$0.14800+0.21313(45)$ & $0.24551(54)$ & $0.07749(54)$ \\
$0.14850+0.16341(58)$ & $0.19043(71)$ & $0.06549(63)$ \\
$0.14900+0.10884(71)$ & $0.12802(83)$ & $0.04702(83)$ \\
$0.14930+0.07927(54)$ & $0.09371(65)$ & $0.03569(79)$ \\
$0.14950+0.06317(70)$ & $0.07495(83)$ & $0.02923(50)$ \\
$0.14980+0.03460(63)$ & $0.04120(74)$ & $0.01552(35)$ \\
$0.15000+0.01702(34)$ & $0.02036(41)$ & $0.00776(17)$ \\
\hline \hline
\end{tabular}

TABLE XI: PCAC mass, chiral condensate, decay constant in six-flavor theory on $L / a=32$ and $\beta=2.0$ lattices. All lattices has the same geometry $32^{3} \times 64$.

\begin{tabular}{cccc}
\hline \hline$\kappa$ & $2 a m_{\mathrm{PCAC}}$ & $a^{3}\langle\bar{\psi} \psi\rangle_{\text {subt }}$ & $a f_{P}$ \\
\hline $0.14850+0.16108(20)$ & $0.18773(23)$ & $0.06225(45)$ \\
$0.14920+0.09165(25)$ & $0.10835(29)$ & $0.04138(69)$ \\
$0.14965+0.04672(35)$ & $0.05567(41)$ & $0.02326(32)$ \\
$0.14980+0.03239(19)$ & $0.03868(22)$ & $0.01582(23)$ \\
\hline \hline
\end{tabular}

\section{A. meson masses}

The lattice action employed in this work depends on three parameters, the number $l=$ $L / a$ of sites along spatial direction, the inverse of the bare gauge coupling constant $\beta=4 / g_{0}^{2}$, and the hopping parameter $\kappa$. For each set of lattice parameters, the (bare) PCAC quark mass in unit of the lattice spacing $a, a m_{\mathrm{PCAC}}$, is obtained as described in Sec. IIC. Figure 21 
TABLE XII: Meson masses in six-flavor theory on $L / a=16$ and $\beta=2.0$ lattices.

\begin{tabular}{ccccc}
\hline \hline$\kappa$ & $a M_{P}$ & $a M_{V}$ & $a M_{S}$ & $a M_{A}$ \\
\hline 0.13800 & $1.5152(51)$ & $1.5388(53)$ & $2.270(60)$ & $2.237(48)$ \\
0.14100 & $1.3213(52)$ & $1.3543(75)$ & $1.908(61)$ & $1.926(50)$ \\
0.14300 & $1.1551(34)$ & $1.1845(51)$ & $1.701(69)$ & $1.718(65)$ \\
0.14500 & $0.9347(16)$ & $0.9412(23)$ & $1.321(17)$ & $1.323(13)$ \\
0.14600 & $0.8086(16)$ & $0.8391(19)$ & $1.111(26)$ & $1.145(23)$ \\
0.14700 & $0.6587(32)$ & $0.6871(46)$ & $0.871(34)$ & $0.868(22)$ \\
0.14750 & $0.5813(29)$ & $0.6110(34)$ & $0.765(18)$ & $0.777(21)$ \\
0.14760 & $0.5755(27)$ & $0.6079(35)$ & $0.745(11)$ & $0.754(11)$ \\
0.14770 & $0.5674(17)$ & $0.6045(19)$ & $0.752(27)$ & $0.763(22)$ \\
0.14785 & $0.5679(29)$ & $0.6034(31)$ & $0.6792(92)$ & $0.707(12)$ \\
0.14800 & $0.5574(27)$ & $0.5950(33)$ & $0.645(12)$ & $0.6777(72)$ \\
0.14900 & $0.5404(26)$ & $0.5761(28)$ & $0.5571(35)$ & $0.5913(40)$ \\
0.14950 & $0.5427(29)$ & $0.5812(30)$ & $0.5539(31)$ & $0.5909(31)$ \\
\hline \hline
\end{tabular}

summarizes $a m_{\mathrm{PCAC}}$ as a function of $1 / \kappa$ at each lattice size. It shows that $a m_{\mathrm{PCAC}}$ does not sustain visible finite size effect and is thus a short-distance quantity. Moreover, it depends on $1 / \kappa$ monotonically. It thus enables to convert the dependence of various observables on $\kappa$ to that on $a m_{\mathrm{PCAC}}$, which is often referred to as the "quark mass dependence" somewhat loosely.

Figure 22 summarizes the masses of the lightest mesons in the (flavor-nonsinglet) pseudoscalar and vector channels. The numerical values of meson masses are tabulated in Tabs. XII, XIII and XIV. For a fixed lattice size, the meson masses are seen to be bounded from below, and cannot approach to zero even for $m_{\mathrm{PCAC}} \rightarrow 0$. The bounds on meson masses depend on the sizes of lattices and are smaller for larger size of lattice. Moreover, the meson mass at $l_{1}$ is compatible with that at $l_{2}>l_{1}$ for the quark mass above some $a m_{\mathrm{PCAC}}$, but below it the change of the former slows down and branches off from those of larger sizes of lattices. This phenomenon is hence considered to be caused by the finite size effect. That is, the finite size effect acts to increase the meson masses, and it eventually prevents them to decrease further for quark mass smaller than some value. 
TABLE XIII: Meson masses in six-flavor theory on $L / a=24$ and $\beta=2.0$ lattices.

\begin{tabular}{ccccc}
\hline \hline$\kappa$ & $a M_{P}$ & $a M_{V}$ & $a M_{S}$ & $a M_{A}$ \\
\hline 0.14100 & $1.319(3)$ & $1.346(3)$ & $1.814(74)$ & $1.789(51)$ \\
0.14300 & $1.154(3)$ & $1.184(3)$ & $1.628(44)$ & $1.675(39)$ \\
$0.14400 \mathrm{P}$ & $1.050(3)$ & $1.079(4)$ & $1.476(28)$ & $1.489(37)$ \\
0.14500 & $0.9400(34)$ & $0.9702(44)$ & $1.273(21)$ & $1.228(65)$ \\
0.14600 & $0.8105(44)$ & $0.8401(58)$ & $1.005(54)$ & $1.067(59)$ \\
0.14700 & $0.6554(19)$ & $0.6847(26)$ & $0.926(18)$ & $0.939(12)$ \\
0.14800 & $0.4801(16)$ & $0.5057(19)$ & $0.646(20)$ & $0.659(17)$ \\
0.14850 & $0.4035(21)$ & $0.4334(29)$ & $0.5857(77)$ & $0.5907(54)$ \\
0.14900 & $0.3472(52)$ & $0.3660(58)$ & $0.376(11)$ & $0.423(13)$ \\
0.14930 & $0.3592(49)$ & $0.3799(73)$ & $0.3756(46)$ & $0.4003(91)$ \\
0.14950 & $0.3631(28)$ & $0.3973(28)$ & $0.3754(35)$ & $0.4088(28)$ \\
0.14980 & $0.3657(30)$ & $0.4064(38)$ & $0.3711(40)$ & $0.4129(53)$ \\
0.15000 & $0.3588(37)$ & $0.3960(28)$ & $0.3612(37)$ & $0.3990(30)$ \\
\hline
\end{tabular}

TABLE XIV: Meson masses in six-flavor theory on $L / a=32$ and $\beta=2.0$ lattices.

\begin{tabular}{ccccc}
\hline \hline$\kappa$ & $a M_{P}$ & $a M_{V}$ & $a M_{S}$ & $a M_{A}$ \\
\hline 0.14850 & $0.3808(10)$ & $0.4044(11)$ & $0.4977(56)$ & $0.5333(76)$ \\
0.14920 & $0.2755(23)$ & $0.3019(21)$ & $0.3542(47)$ & $0.3698(50)$ \\
0.14965 & $0.2510(26)$ & $0.2783(30)$ & $0.2658(26)$ & $0.2933(29)$ \\
0.14980 & $0.2719(17)$ & $0.2990(24)$ & $0.2759(17)$ & $0.3040(28)$ \\
\hline \hline
\end{tabular}

The finite size effect observed above in the meson masses in our target system is qualitatively different from that in $\mathrm{SU}(2)_{\mathrm{C}}$ gauge theory with two adjoint Dirac fermions [27, 60], where the pseudoscalar meson mass is smaller for smaller size of lattice. Tables XII, XIII and XIV also show that the pseudoscalar meson becomes degenerate in mass with the scalar meson once the decrease of the meson masses almost stops. The finite size effect on the meson masses in our target system resembles that obtained in the results of the simulation for $N_{F}=2$ system at very weakly coupling $(\beta=4.0)$ and finite volume in Sec. III,

Another noteworthy feature found in Fig. 22 is the approximate degeneracy of the vector 


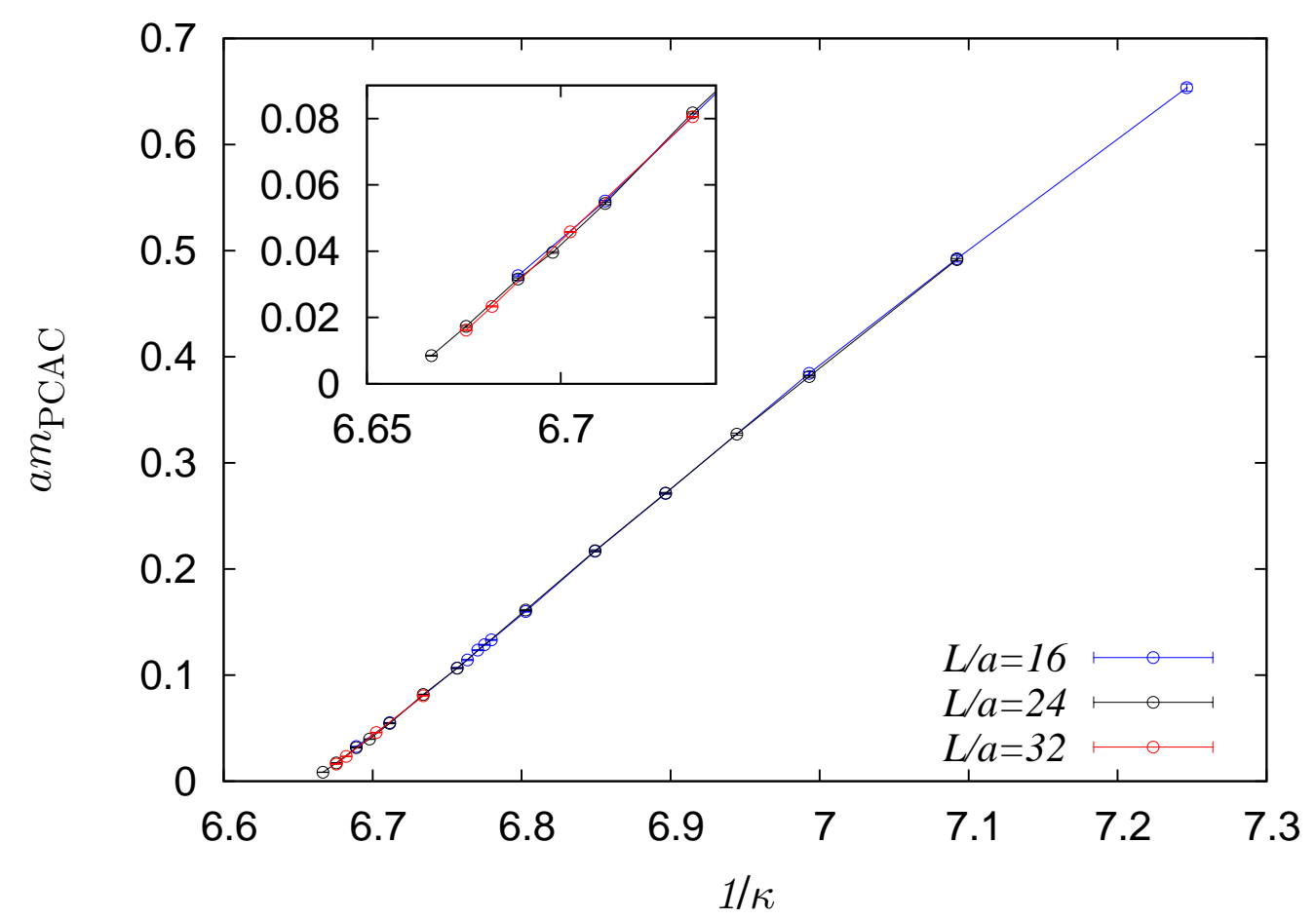

FIG. 21: $a m_{\mathrm{PCAC}}$ versus the inverse of the hopping parameter in six-flavor theory.

meson with the pseudoscalar meson in mass. This fact prevents us to try to fit the data using chiral perturbation theory. The approximate degeneracy in mass between pseudoscalar meson and vector meson at first sight reminds us of the heavy quark limit in which they form a multiplet. If the quark is heavy enough, the meson mass should vary almost linearly. However, Fig. 22 shows that this possibility is unlikely. To examine this point more closely, the ratio

$X_{1 / 2} \equiv \frac{a M_{P}}{\left(a m_{\mathrm{PCAC}}\right)^{1 / 2}}$,

is plotted in Fig. 23. In what follows, the dependence of $a$ on the quark mass is assumed to be small enough that it does not to affect to the observation. From Fig. 23, $a M_{P}$ seems to vary with the power 0.5 in the region $a m_{\mathrm{PCAC}} \gtrsim 0.35$. The ratio $X_{1 / 2}$ in that figure starts to increase at some quark mass depending on the size of lattice, and then diverges for $m_{\mathrm{PCAC}} \rightarrow 0$, since $M_{P}$ eventually stops to decrease at finite volume. We consider that the results for such parameters offer no knowledge on the dynamics of the theory.

Rather, the most important point to notice is as follows; if we look at the data toward smaller quark mass, the part of $X_{1 / 2}$ that do not suffer finite volume correction so much 


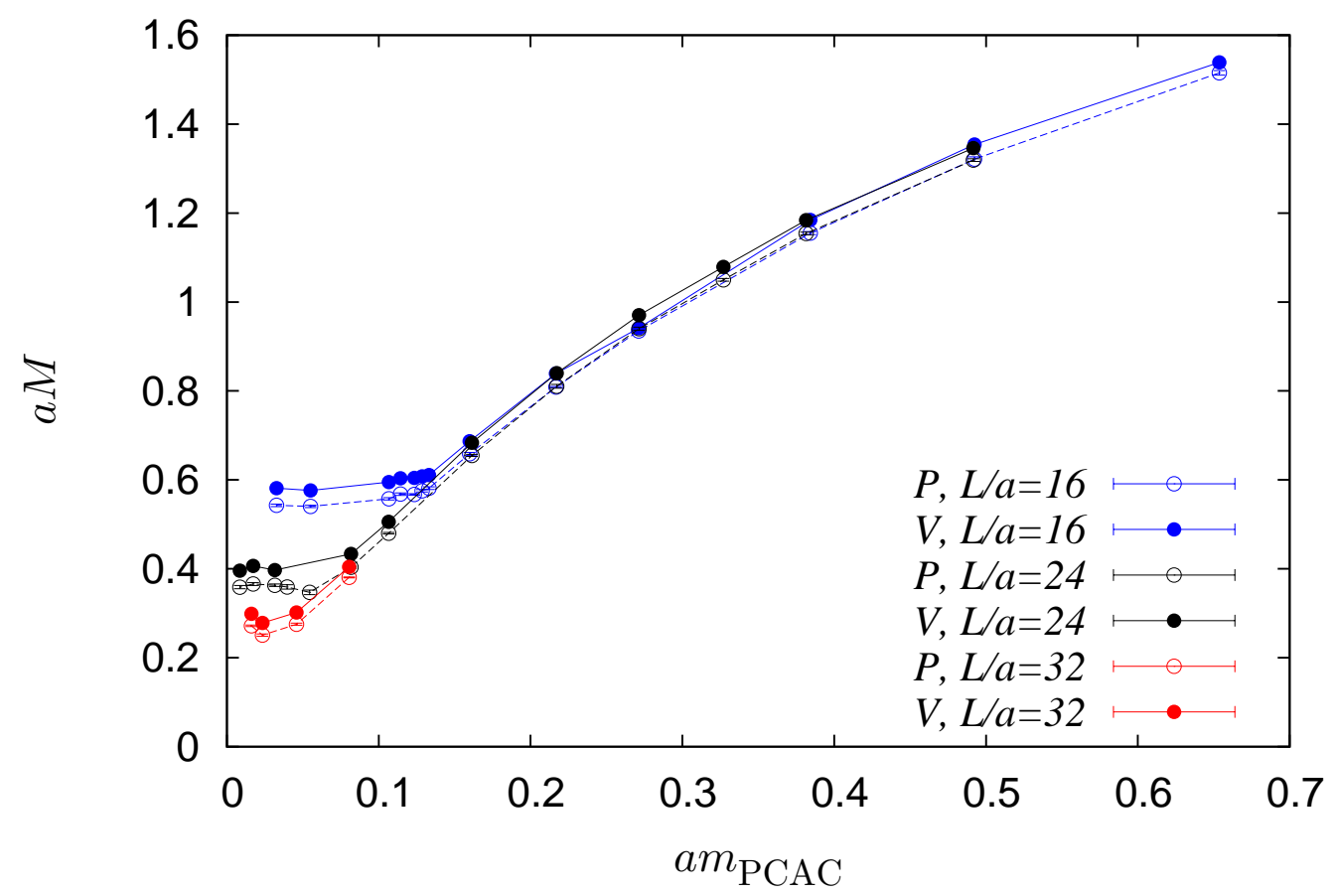

FIG. 22: The lightest pseudoscalar meson mass $a M_{P}$ (open dot) and the lightest vector meson mass $a M_{V}$ (filled dot) versus $a m_{\mathrm{PCAC}}$ in six-flavor theory. The individual lines are drawn just to guide eyes.

decrease in the region $a m_{\mathrm{PCAC}} \lesssim 0.3$. If the chiral symmetry breaking is realized in the system, $M_{P}$ should be better approximated by $\left(m_{\mathrm{PCAC}}\right)^{1 / 2}$ for lighter quarks. As a reference, we show $X_{1 / 2}$ simulated for the two-flavor theory at $\beta=2.0$ in Fig. 24. In contrast to it, in the six-flavor theory, $a M_{P} \propto\left(a m_{\mathrm{PCAC}}\right)^{1 / 2}$ for $0.35<a m_{\mathrm{PCAC}}<0.65$, but it changes with different exponent, $a M_{P} \propto\left(a m_{\mathrm{PCAC}}\right)^{\alpha}$ with $\alpha>0.5$, at smaller quark mass. This is the first evidence supporting the absence of chiral symmetry breaking in the six-flavor theory.

Figure 23 shows that available data are all in the transition region over which the exponent $\alpha_{M_{P}}$ changes from 0.5 to that in the basin of the IR-fixed point. Moreover, the scaling $M_{P} \propto\left(m_{\mathrm{PCAC}}\right)^{\alpha_{M_{P}}, \star}$ with $\alpha_{M_{P}, \star}=1 /\left(1+\gamma_{\star}\right)$ predicted according to the hyperscaling hypothesis [27, 28] implies that $1>\alpha_{M_{P}, \star}>0.5$ for $0<\gamma_{\star}<1$. Unless the data at very small quark mass $\lesssim 0.01$ are available, the fit to determine the term $c_{M_{P}}\left(a m_{\mathrm{PCAC}}\right)^{\alpha_{M_{P}}}$ just looks at the height of the data, but poorly captures the bent of the curve, implying no hope for reliable estimate of $\alpha_{M_{P}}$. Here, we are just content with observing $X_{\alpha} \equiv a M_{P} /\left(a m_{\mathrm{PCAC}}\right)^{\alpha}$ 


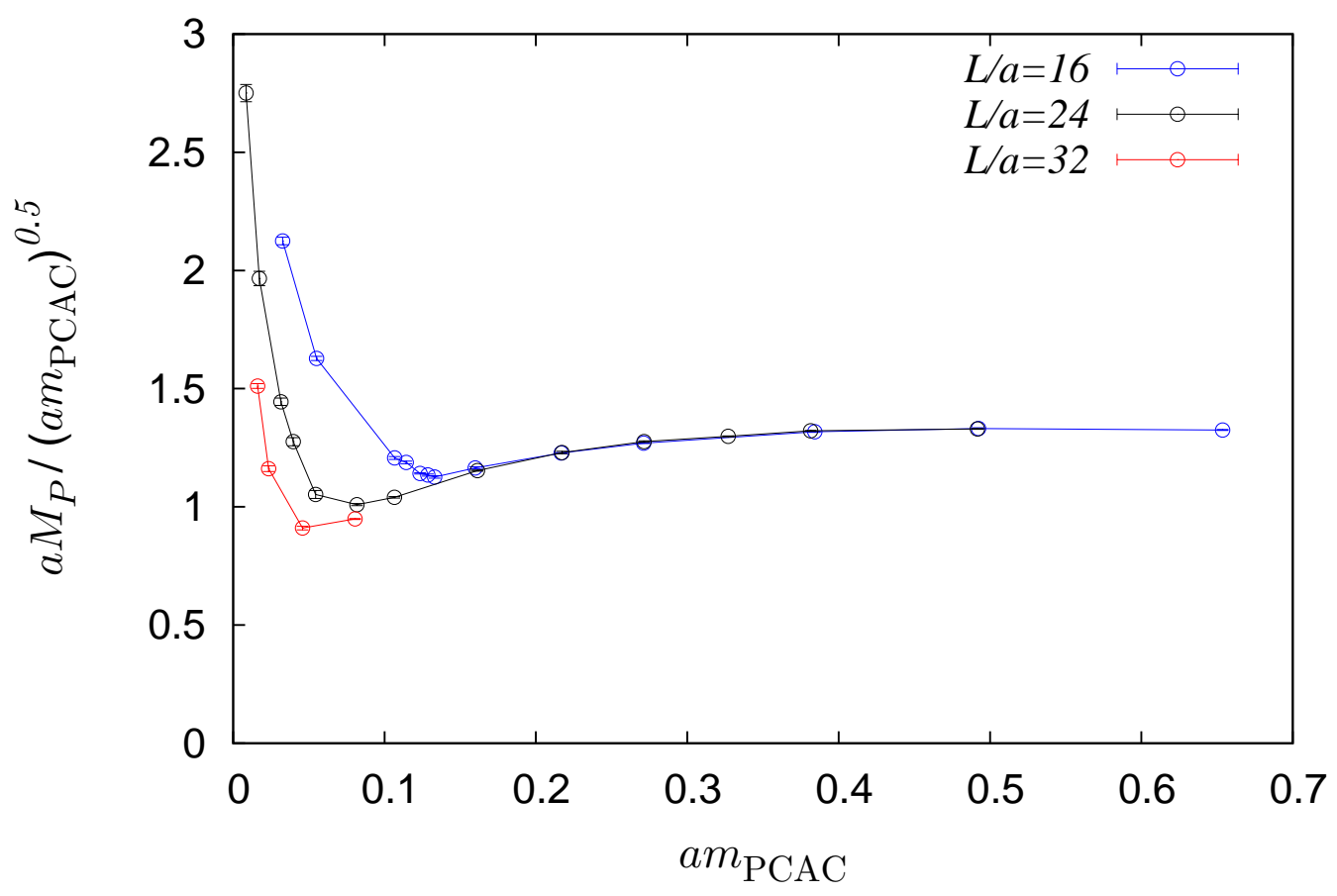

FIG. 23: The quark mass dependence of $X_{1 / 2}=a M_{P} /\left(a m_{\mathrm{PCAC}}\right)^{1 / 2}$ in six-flavor theory. $X_{1 / 2}$ decreases below $a m_{\mathrm{PCAC}} \lesssim 0.3$ until the finite size effect shows up.

for $\alpha=0.66(\gamma \equiv 1 / \alpha-1 \simeq 0.52)$ and $\alpha=0.73(\gamma \simeq 0.3)$ in Figs. 25] and 26, respectively. Those figures indicate that the exponent $\alpha_{M_{P}}$ suggested in the present spectroscopy study at $\beta=2.0$ is compatible with that found in the study of the running coupling constant defined in the Schrödinger functional scheme

$0.26 \leq \gamma_{\star, \mathrm{SF}} \leq 0.74$

As discussed in Sec. IIE, the spectrum and the dynamics at low energy, which could also be affected by the representations of fermions, determine the qualitative behavior of the finite size effect. It is thus necessary to scrutinize the dependence of other observables, such as $f_{P}$ and the subtracted chiral condensate, on quark masses and lattice sizes.

\section{B. decay constant}

Figure 27 shows the quark mass dependence of the decay constant $f_{P}$ of the lightest pseudoscalar meson $P$ in unit of the lattice spacing $a$ obtained through Eq. (9). There, 


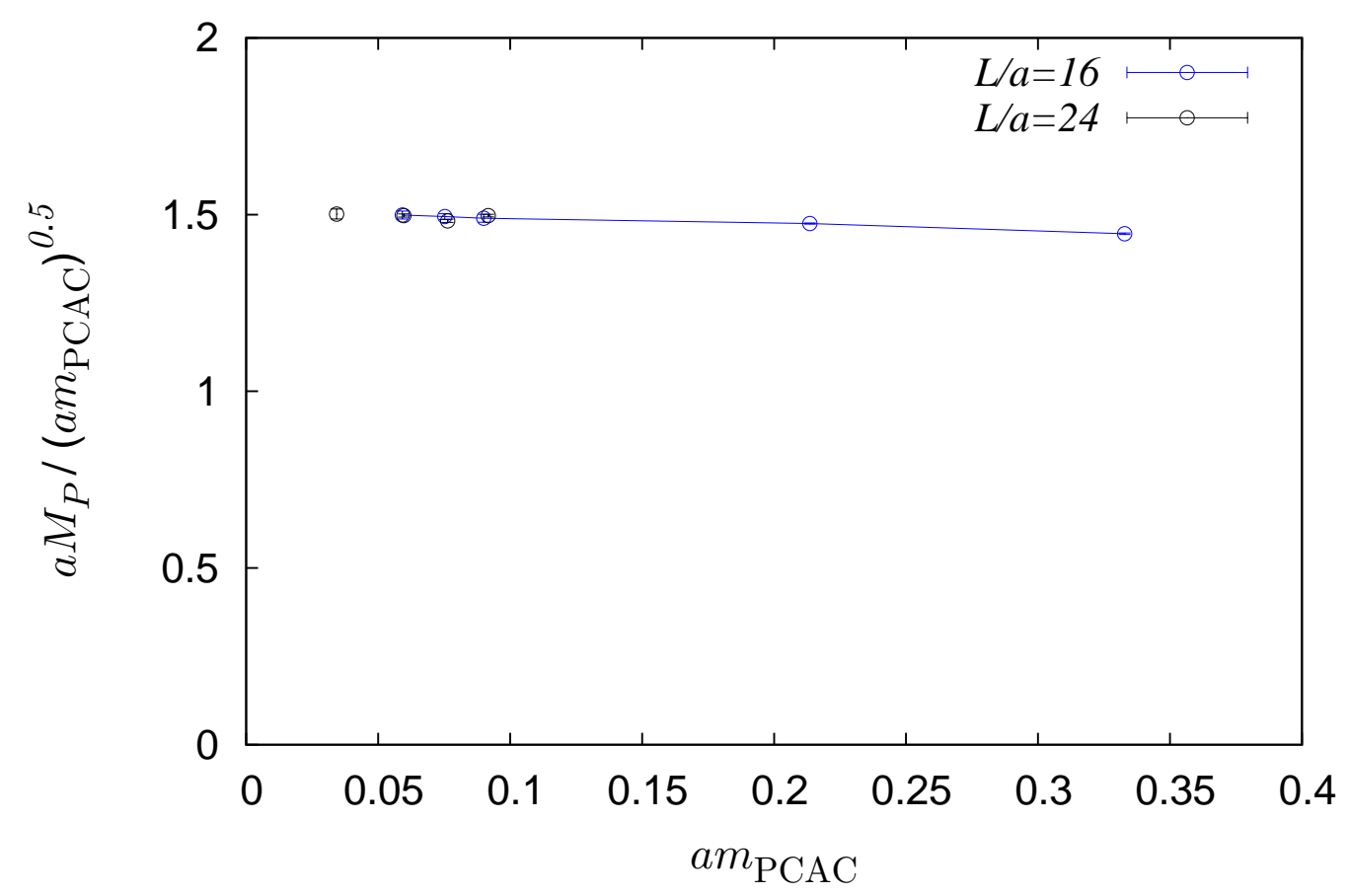

FIG. 24: The quark mass dependence of $a M_{P} /\left(a m_{\mathrm{PCAC}}\right)^{1 / 2}$ in two-flavor theory.
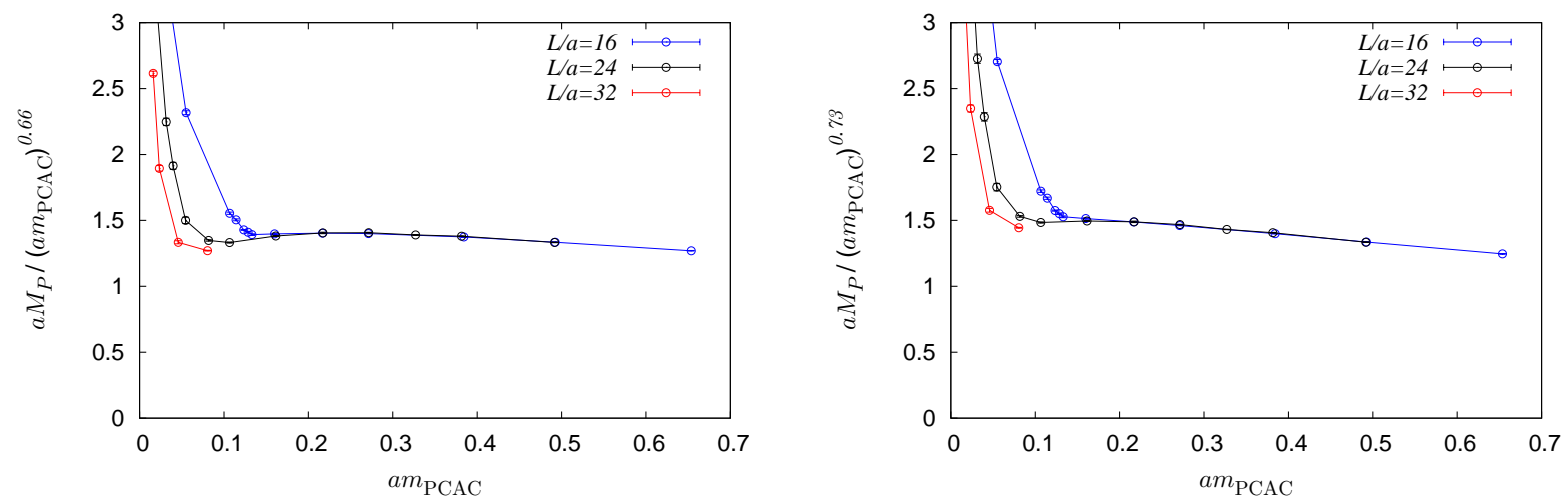

FIG. 25: The quark mass dependence of FIG. 26: The quark mass dependence of $a M_{P} /\left(a m_{\mathrm{PCAC}}\right)^{\alpha}$ with $\alpha=0.66$ in six-flavor $a M_{P} /\left(a m_{\mathrm{PCAC}}\right)^{\alpha}$ with $\alpha=0.73$ in six-flavor theory. theory.

$f_{P}$ appears to vanish in the chiral limit, $m_{\mathrm{PCAC}} \rightarrow 0$. However, this seems to originate from the bounded behavior of the pseudoscalar meson mass $M_{P}$ in the chiral limit at finite volume: Eq. (9) implies that $f_{P}$ will vanish unless $M_{P}$ approaches to zero for $m_{\mathrm{PCAC}} \rightarrow 0$. 


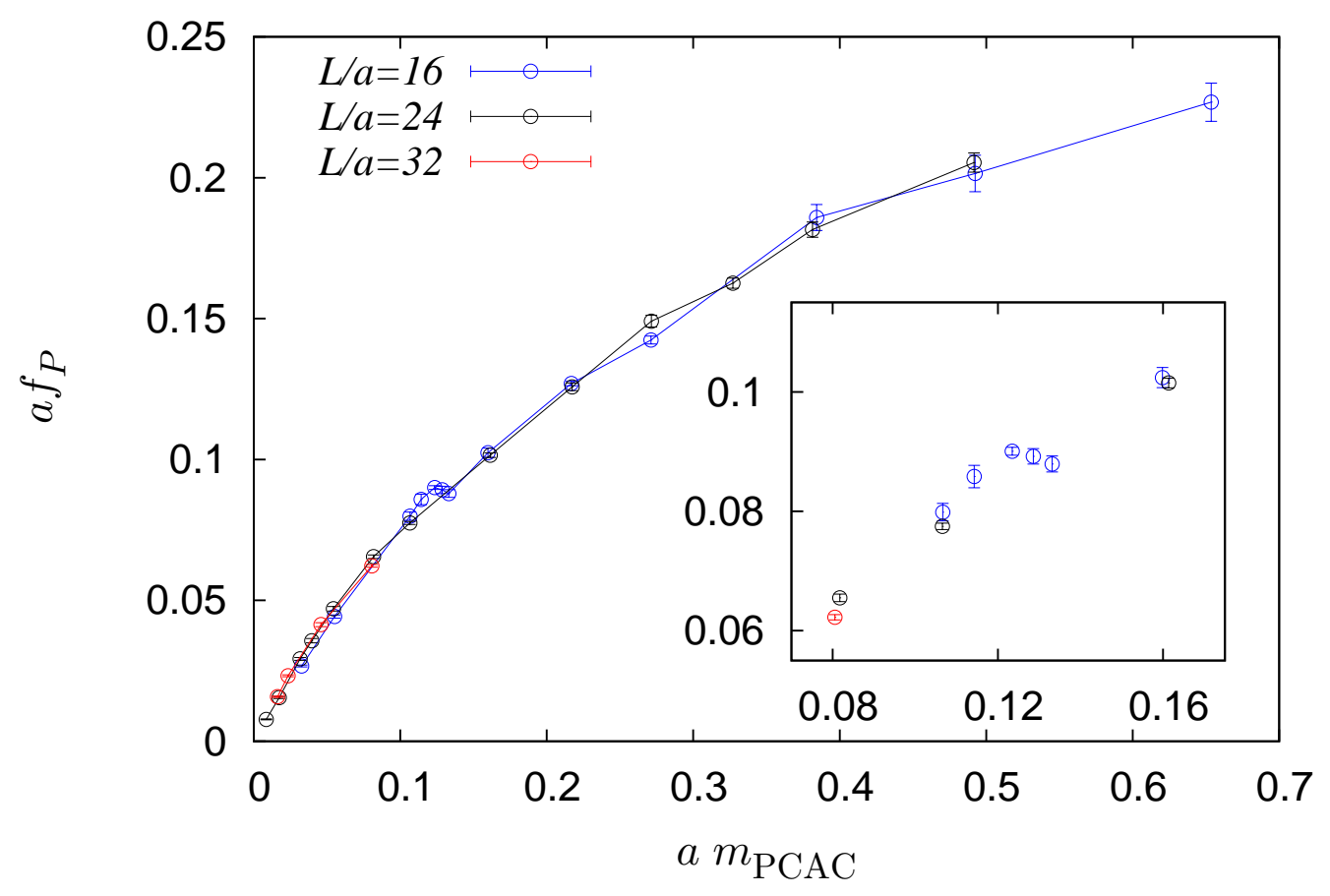

FIG. 27: The quark mass dependence of $a f_{P}$ in six-flavor theory.

The analysis of the data shows that the factor $A_{P P}$ in Eq. (6) related to the wave function of $P$ is slightly more sensitive to the finite size effect than $M_{P}$. Once $M_{P}$ almost ceases to decrease, $a f_{P}$ starts to vanish almost linearly in $a m_{\mathrm{PCAC}}$. Actually, we saw in Sec. III that the same phenomenon happens even in the two-flavor theory at very weakly coupling ( $\beta=4.0)$. It is caused by the finite size effect. These imply that we cannot find anything about dynamics in the quark mass region where meson masses cease to decrease at each lattice size $\left(L \ll 1 / M_{P}\right.$ in our case).

We rather focus on the behavior of $f_{P}$ around the quark mass where the finite size effect begins to show up but the meson masses do not cease to decrease. For the lattice size $L / a=16$, Fig. 22 implies that $a M_{P}$ begins to suffer visible finite size effect around $a m_{\mathrm{PCAC}}=0.12 \sim 0.14$. The simulation has thus been done for many parameters in this region. Fig. 27 shows that the finite size effect acts to increase $a f_{P}$ in this region. The difference between $L / a=24$ and $L / a=32$ found at $a m_{\mathrm{PCAC}} \simeq 0.08$ is also compatible with such a tendency. This behavior is opposite to that in Fig. 2 expected for the finite size effect in the theory with chiral symmetry breaking. The behavior of $L / a=16$ data suggests the 
example 4 in Fig. 6 for the finite size effect on $a f_{P}$. This difference in the features of the finite size effect implies that the six-flavor is unlikely a $\chi$-theory.

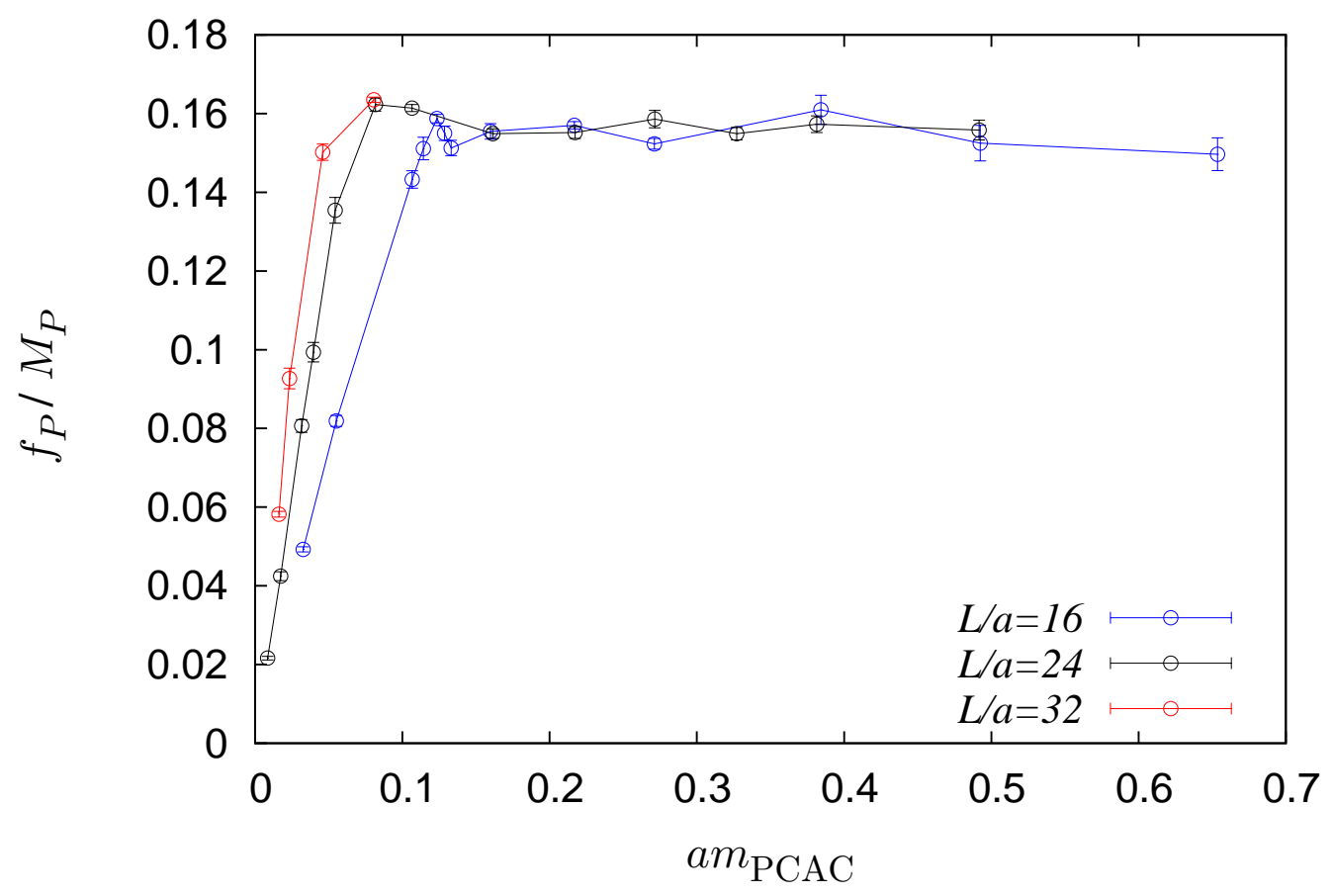

FIG. 28: The quark mass dependence of the ratio $f_{P} / M_{P}$ in six-flavor theory.

We next focus on the ratio $f_{P} / M_{P}$. In the $\chi$-theory, this ratio should blow up in the chiral limit. In the IR-conformal theory, if the hyperscaling hypothesis is valid, $f_{P}$ depends on the quark mass with the same power as $M_{P}$ [27, 28] so that the dependence of their ratio $f_{P} / M_{P}$ on the quark mass will fade away in the scaling region. Figure 28 shows the result in the six-flavor theory. The behavior of $f_{P}$ and $M_{P}$ implies that $f_{P} / M_{P}$ starts to decrease once $M_{P}$ almost stops to decrease at finite volume. We recall that, even in two-flavor theory, the ratio $f_{P} / M_{P}$ seems to start to blow up at far smaller quark mass (Figure 11). The data for $0.13 \lesssim a m_{\mathrm{PCAC}} \lesssim 0.25$ are almost flat, but the data with smaller $a m_{\mathrm{PCAC}}$ leave the possibility for the ratio to increase in the chiral limit. However, the behavior of the data $L / a=16$ around $a m_{\mathrm{PCAC}} \sim 0.13$ suggests the possibility that $f_{P} / M_{P}$ jumps once before starting to drop steeply. To settle this issue, simulation with larger lattices, say, of size $L / a=48$, is required. 


\section{C. chiral condensate}

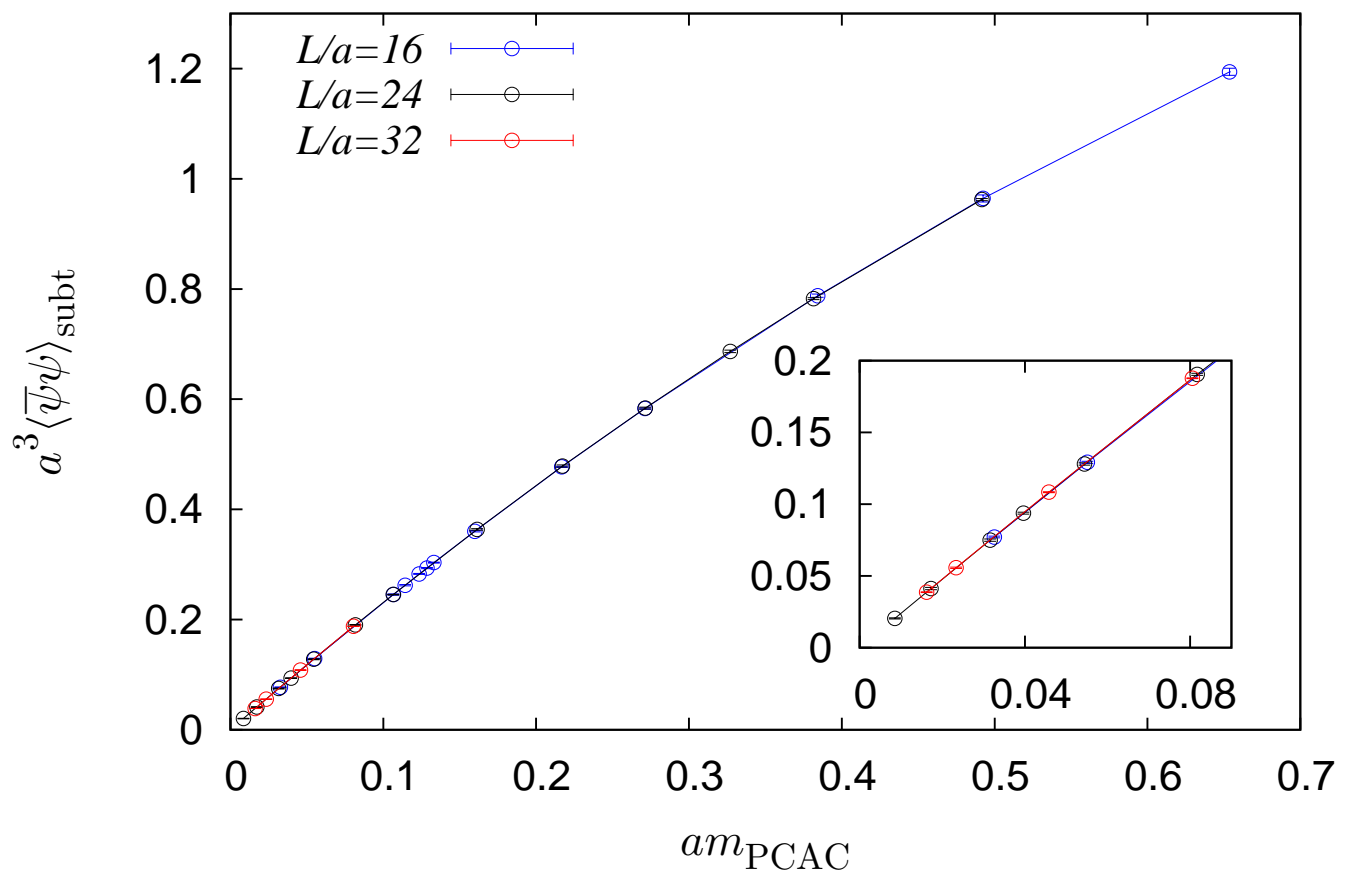

FIG. 29: The subtracted chiral condensate versus $a m_{\mathrm{PCAC}}$ in six-flavor theory. The lines are drawn for the individual sizes of lattices just to guide the eyes.

We next focus on the chiral condensate. We recall the result of the hyperscaling analysis applied to the chiral condensate [28]. In the attractive basin of the IR fixed point, the nonanalytic and universal term with the exponent $\alpha_{\bar{q} q}=\frac{3-\gamma_{\star}}{1+\gamma_{\star}}$ for the mass anomalous dimension $\gamma_{\star}$ is expected to emerge. The analysis thus far indicates that $\gamma_{\star}<1$ provided that the sixflavor theory turns out to be IR-conformal. Then, $\alpha_{\bar{q} q}>1$. Therefore, the analytic term linear in the quark mass dominates the chiral condensate at small quark mass.

Figure 29 shows the result for the subtracted chiral condensate $\langle\bar{\psi} \psi\rangle_{\text {subt }}$ in the six-flavor theory. The lines in that figure are drawn to guide the eyes to the plots of the individual sizes of lattices. The three lines overlap with each other. We stress the non-triviality of this fact, and then make an important observation on the properties of the data of the subtracted chiral condensate, which is crucial for our succeeding analysis. The subtracted chiral condensate $\langle\bar{\psi} \psi\rangle_{\text {subt }}\left(m_{\mathrm{PCAC}}, L / a\right)$ defined in Eq. (11) will diminish more rapidly once 
the finite size effect almost stops decrease of the pseudoscalar meson mass $M_{P}$. With this in our mind, we examine the quark mass and volume dependence of meson masses in Fig. 22 more closely. On one hand, at $L / a=16$, meson masses start to suffer the finite size effect around $a m_{\mathrm{PCAC}} \sim 0.14$, and has already ceased to decrease around $a m_{\mathrm{PCAC}} \sim 0.10$. On the other hand, meson masses at $L / a=32$ and $a m_{\mathrm{PCAC}} \sim 0.08$ do not seem to suffer visible finite size effect. Figure 29] shows that no visible finite size effect is observed for $\langle\bar{\psi} \psi\rangle_{\text {subt }}$ even for the quark mass in this region. This indicates that the finite size effect appears to be negligibly small in $\langle\bar{\psi} \psi\rangle_{\text {subt }}$ over the entire region of available data ${ }^{4}$. Based on this observation, we perform various fits and ask whether the chiral limit is consistent with 0 within available precision.

We consider the following three types of target data to be fit;

(S1) data with $a m_{\mathrm{PCAC}} \leq 0.2$,

(S2) data with $a m_{\mathrm{PCAC}} \leq 0.1$,

(S3) data for which $a m_{\mathrm{PCAC}} \leq 0.2$ and $f_{P} / M_{P}$ does not belong to the sharply falling region in Fig. 28.

No restrictions on lattice sizes are imposed. As the fit functions, the following three will be examined;

(F1) linear function $f_{2}\left(x=a m_{\mathrm{PCAC}}\right)$ in Eq. (12),

(F2) quadratic function $f_{3}\left(x=a m_{\mathrm{PCAC}}\right)$ in Eq. (14),

(F3) function containing a term with indefinite exponent

$$
f_{4}\left(x=a m_{\mathrm{PCAC}}\right)=c_{0}+c_{1} x+c_{2} x^{\alpha} .
$$

As seen in Fig. 29, the shape of the chiral condensate versus quark mass is convex upward. Therefore, the chiral extrapolation by linear fit to the data with negligible finite size effect will tend to overestimate the value in the massless limit. If it turns out to vanish within the available precision, the result will support absence of chiral symmetry breaking. This motivates us to do the linear fit first, but Tab. XV shows that the answer is not affirmative.

\footnotetext{
4 We will examine the effect of the data with very small quark masses on the result deliberately.
} 
TABLE XV: Result for the linear fit to the subtracted chiral condensate, where $a_{0}$ is the value in chiral limit. See the text for the definition of three data data, S1, S2 and S3.

\begin{tabular}{|c|c|c|}
\hline data set & $a_{0}$ & $a_{1}$ \\
\hline $\mathrm{S} 1$ & $0.00406(78)$ & $2.2499(86)$ \\
\hline $\mathrm{S} 2$ & $0.00137(27)$ & $2.3191(58)$ \\
\hline S3 & $0.0131(11)$ & $2.1766(88)$ \\
\hline
\end{tabular}

TABLE XVI: Result for the fit to the subtracted chiral condensate by the quadratic fit, where $b_{0}$ is the value in chiral limit. $\mathbf{S} \mathbf{1}^{\prime}$ is obtained by discarding the data with $a m_{\mathrm{PACA}} \leq 0.03$ in $\mathbf{S 1}$.

\begin{tabular}{cccc}
\hline \hline data set & $b_{0}$ & $b_{1}$ & $b_{2}$ \\
\hline $\mathbf{S 1}$ & $-0.00003(12)$ & $2.4030(35)$ & $-0.927(21)$ \\
$\mathbf{S 2}$ & $0.00017(20)$ & $2.3897(97)$ & $-0.76(10)$ \\
$\mathbf{S 3}$ & $0.00142(76)$ & $2.382(13)$ & $-0.849(53)$ \\
\hline $\mathbf{S 1}^{\prime}$ & $-0.00021(24)$ & $2.4068(59)$ & $-0.947(31)$ \\
\hline \hline
\end{tabular}

Table XVI] shows the result of the fit with the quadratic function (14). The quadratic function consists of the leading and the next-to-leading (NLO) analytic corrections, but with no non-analytic term such as chiral logarithm. We regard it as being one of the trial functions to examine the consistency of the presence of the chiral symmetry breaking. The intercept $b_{0}$ at $m_{\mathrm{PCAC}}=0$ is non-vanishing for the fit to the data set $\mathbf{S 3}$, but the smallest $a M_{P}$ in $\mathbf{S 3}$ is about 0.38 there. From the above observation about the smallness of the finite size effect on the data of the subtracted chiral condensate $\langle\bar{\psi} \psi\rangle_{\text {subt }}$, there is no reason to disregard the results for the data sets, S1 and S2. Figure 30 shows how the fit curves pass through the data in S1. One notices that the data with small quark masses are slightly separated from the line determined by the linear fit. In order to examine the significance of the data with relatively small quark mass in the quadratic fit, we intentionally remove the data with $a m_{\mathrm{PCAC}} \leq 0.03$ from $\mathbf{S 1}$, and carry out the quadratic fit. As shown in Figure 31, the result does not exhibit essential change. (The coefficients are listed as $\mathbf{S} \mathbf{1}^{\prime}$ in Tab. XVI.) This indicates that the difference in the chiral limit between the two functions is not attributed to those data with relatively small quark mass. Rather, Tab. XVI shows the difference in the coefficient of the NLO term, $b_{2}$, between $\mathbf{S 1}$ and $\mathbf{S 2}$, indicating that $b_{2}$ is mostly determined 


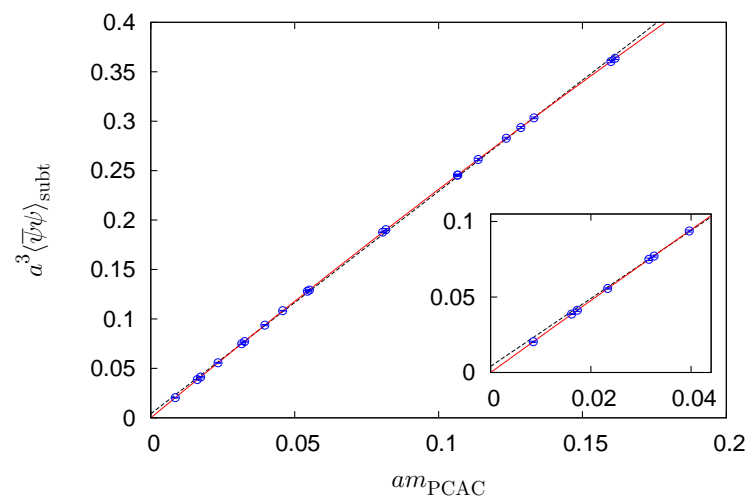

FIG. 30: Result for the fit to the data set S1 by the linear function (dotted line) and quadratic function (red curve). Only the fit curves with the central values for the coefficients are drawn.

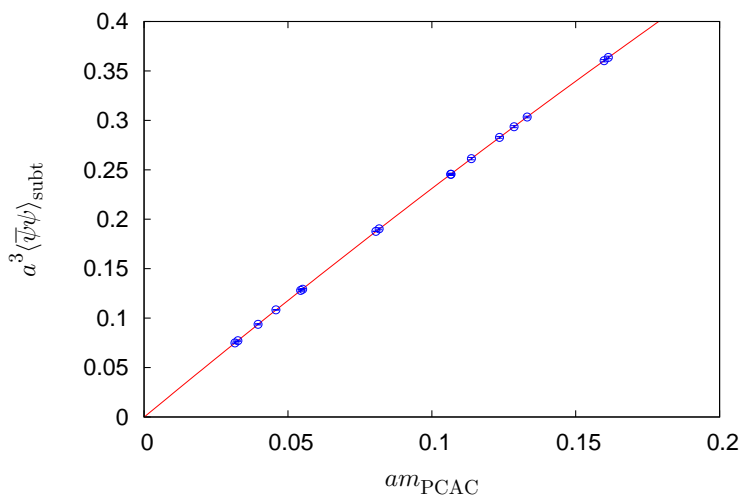

FIG. 31: Result for the quadratic fit to the data set $\mathbf{S} \mathbf{1}^{\prime}$ obtained from the data set $\mathbf{S} \mathbf{1}$ by discarding the data with $a m_{\mathrm{PCAC}} \leq 0.03$.

by the subset of data with larger quark mass in each set, which is thought to suffer far smaller finite size effect. With $b_{2}$ determined as such, $\langle\bar{\psi} \psi\rangle_{\text {subt }}$ in the limit $m_{\mathrm{PCAC}} \rightarrow 0$ is found to be consistent with zero both for $\mathbf{S} 1$ and $\mathbf{S 2}$ within the precision of data available at present.

TABLE XVII: Result for fit to the subtracted chiral condensate by the function (21) with the exponent $\alpha$ to be determined. $c_{0}$ is the value in chiral limit.

\begin{tabular}{ccccc}
\hline \hline data set & $c_{0}$ & $c_{1}$ & $c_{2}$ & $\alpha$ \\
\hline S1 & $0.00002(22)$ & $2.398(15)$ & $-0.96(12)$ & $2.04(12)$ \\
S2 & $-0.00023(79)$ & $2.48(31)$ & $-0.401(55)$ & $1.39(80)$ \\
S3 & $0.001(11)$ & $2.38(38)$ & $-0.845(93)$ & $2.0(1.8)$ \\
\hline \hline
\end{tabular}

Table XVII is the result for the fit with the indefinite exponent (21). For the data set S1, the exponent is found to be nearly equal to 2 and the values of the other parameters coincide with those in Tab. XVI obtained by the quadratic fit. The fit with the function (21) contains four parameters to be determined, and the values of the parameters are contaminated by the uncertainty larger than that in the quadratic fit. Nevertheless, the intercept $c_{0}$ is consistent with zero. This motivates us to carry out the fit with the function (21) but now with $c_{0}$ 
constrained to 0, i.e. assuming IR-conformality, and try to examine if the resulting exponent is consistent with that (20) found in Schrödinger functional scheme.

As remarked above, the chiral condensate has a non-analytic and universal piece $\left(m_{q}\right)^{\alpha_{\bar{q} q}}$ with the exponent $\alpha_{\bar{q} q}=\frac{3-\gamma_{\star}}{1+\gamma_{\star}}$ in the mass-deformed IR-conformal theory with hyperscaling hypothesis, and it is sub-dominant as long as the inequality $1<\alpha_{\bar{q} q}<2$ is satisfied (Recall the presence of the next-leading-order analytic term $\propto\left(m_{\mathrm{PCAC}}\right)^{2}$.). As pointed out in Sec. $\mathrm{VA}$, the exponent $\alpha_{M_{P}, \star}=1 /\left(1+\gamma_{\star}\right)$ appearing in the scaling of the pseudoscalar meson mass $M_{P}$ is smaller than 1 if $0<\gamma_{\star}<1$, and the determination of $\alpha_{M_{P}}$ requires the data at very small quark masses. In contrast, it may be possible to give very rough estimation on $\alpha_{\bar{q} q}(>1)$ by performing the fit with the function (21) the set including the data at the quark mass that is modest but belongs to the basin of IRFP. Meanwhile, we observed in Fig. 23 that the exponent may be still in the process of changing towards the value at the infrared fixed point. Thus, we perform the fit with the function (21) under the constraint $c_{0}=0$ only for the set $\mathbf{S} 2$ consisting of data with $a m_{\mathrm{PCAC}}<0.1$, and get

$c_{1}=2.420(31), \quad c_{2}=-0.46(20), \quad \alpha=1.65(30)$.

The above value of the exponent $\alpha$ is not inconsistent with $1.3 \leq \alpha_{\bar{q} q \text {, SF }} \leq 2.2$ corresponding to $\gamma_{\star}$ in Eq. (20), which was obtained by the calculation in the Schrödinger functional scheme.

Finally, Fig. 32 shows the quark mass dependence of $B$ defined by Eq. (16) in the sixflavor theory. We have seen that it decreases and approaches to a non-vanishing value in the chiral limit in the two-flavor theory (Fig. 9). In the six-flavor theory, it increases as quark mass decreases, even if the focus is restricted to the range, $a m_{\mathrm{PCAC}} \gtrsim 0.08$, in which the finite size effect is not substantial. This is discriminated from the behavior of $B$ in Fig. 17 obtained for the two-flavor theory at weak coupling, where the increase is caused by the large finite size effect. Since $\langle\bar{\psi} \psi\rangle \propto m_{q}$ due to dominance of analytic term, and $f_{P}^{2} \propto m_{q}^{2 /\left(1+\gamma_{\star}\right)}$ if the hyperscaling hypothesis holds in this system, $B \propto m_{q}^{-\left(1-\gamma_{\star}\right) /\left(1+\gamma_{\star}\right)}$. Thus, the qualitative behavior found in Fig. 32 is compatible with the scaling with $\gamma_{\star}<1$. 


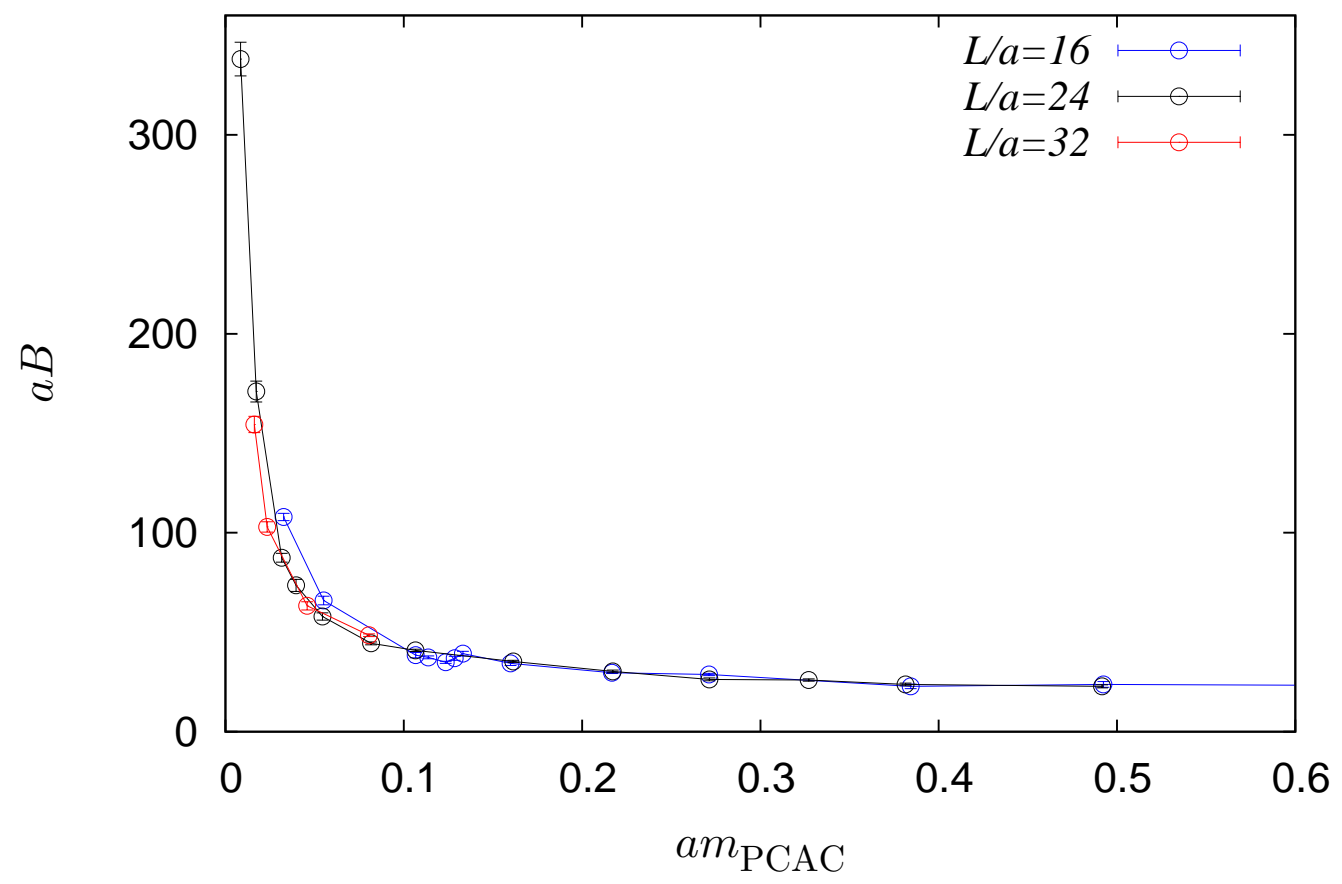

FIG. 32: $B$ versus $a m_{\mathrm{PCAC}}$ in six-flavor theory. 


\section{SUMMARY AND DISCUSSION}

To study the quantum-mechanical dynamics of the $\mathrm{SU}(2)_{\mathrm{C}}$ gauge theory with six Dirac fermions in the fundamental representation, we perform simulation on the lattices with a fixed bare gauge coupling constant $(\beta=2.0)$ and linear size up to $L / a=32$, and present the first results. In this work we use Wilson fermion with no $O(a)$ improvement, leaving the influence of the lattice fermions on the chiral property as a subject to be investigated in the future work.

We first observe that the finite size effect on the meson masses turns out to be substantial and to put the lower bound on masses at each size of lattice. To know the quantitative dependence of the lightest pseudoscalar meson mass $M_{P}$ on the quark mass, it is inevitable to carry out larger lattice simulation such as $L / a=48$. Nevertheless, we have seen that

the data with quark mass $a m_{\mathrm{PCAC}} \gtrsim 0.35$ exhibit the dependence as $M_{P} \propto\left(m_{\mathrm{PCAC}}\right)^{0.5}$, but tends to change more rapidly for smaller quark mass. In the theory with chiral symmetry breaking, the exponent $\alpha_{M_{P}}$ in $M_{P} \propto\left(m_{\mathrm{PCAC}}\right)^{\alpha_{M_{P}}}$ should be closer and closer to 0.5 for smaller and smaller quark mass. This point supports that the theory is an IR-conformal theory with the exponent $\alpha_{M_{P}}$ larger than 0.5.

With help of the explicit simulation of the two-flavor theory, we demonstrate the utility of the subtracted chiral condensate $\langle\bar{\psi} \psi\rangle_{\text {subt }}$ defined by Eq. (11) as a quantity which may help to examine the occurrence of chiral symmetry breaking in the Wilson fermion simulation. We discuss that the finite size effect is negligibly small in all available data of $\langle\bar{\psi} \psi\rangle_{\text {subt }}$. With this observation, the chiral extrapolation is performed and its massless limit is seen to be compatible with 0 within the precision of the available data.

We focus on the qualitative feature of finite size effect on the decay constant $f_{P}$, and search its possible difference from the theory with chiral symmetry breaking. From close examination of data simulated for the six-flavor theory, the finite size effect seems to increase $f_{P}$, opposite to that in the $\not$-theory. We conjecture example 4 shown in Fig 6 is realized in the $N_{F}=6$ theory.

At present, the proposed utility of the finite size effect on $f_{P}$ has a loophole. We recall that the qualitative feature seen in Fig. 2 for the finite size effect on $f_{P}$ is obtained according to the chiral perturbation theory. At present, it is uncertain if the decreasing tendency of $f_{P}$ persists even in the circumstance outside the applicability of chiral perturbation, i.e. on the 
space with too small volume compared with the dynamical length scale. Naively speaking, it is plausible because, if the finite size effect is assumed to tend to increase $f_{P}$ at such weak coupling that chiral perturbation is not applicable, there must be some transition region of parameters where the finite size effect accidentally disappears. The issue could be checked by carrying out the simulation at weak coupling. We attempted to do that at $\beta=4.0$ in the two-flavor theory, but failed to observe a plateau in the effective mass plot in the analysis of $M_{P}$, which is necessary to get $f_{P}$ through the PCAC relation, at $\kappa=0.128$ and $L / a=24$. On the other hand, $M_{P}$ could be determined on the lattice with $\kappa=0.128$ and $L / a=16$. Thus, $\beta=4.0$ may be too weak for the contribution of the excited states to decouple unless the quark mass is so small that the meson masses reach the lower bounds caused by the finite size effect. This issue is left as one of the questions to be settled in order for the finite size effect on $f_{P}$ to become a device to judge the occurrence of chiral symmetry breaking. Meanwhile, we note that, in the six-flavor, the plateaus can be observed in the effective mass plots in all range of measured quark mass, which indicate the distinction from the two-flavor theory, i.e. the system with the chiral symmetry breaking, at very weak coupling.

The seemingly flatness of the ratio $f_{P} / M_{P}$ is also compatible with the IR-conformal theory and the scaling predicted from the hyperscaling hypothesis. At present, we leave the possibility that this ratio may start to blow up at smaller $M_{P}$ for the following two reasons. In Fig. 33, we show $f_{P} / M_{P}$ derived from the data found in the work by Fodor et al. [23] for $\mathrm{SU}(3)_{\mathrm{C}}$ gauge theory with $N_{F}=12$ Dirac fermions. The finite size effect appears there. In particular, at the smallest quark mass, $a m_{q}=0.01$, the data with size $L / a=48$ can be seen to play an important role. However, it is still uncertain if $f_{P} / M_{P}$ starts to blow up as in Fig. 11, while the finite size effect acts to decrease $f_{P}$ in Ref. [23], which is the same tendency as in the $\chi$-theory. We are expecting to report the result for $f_{P} / M_{P}$ obtained using larger lattices in the future.

Another reason is that, although we show here that the chiral symmetry breaking does not seem to occur in the six-flavor theory, it was demonstrated in our preliminary report [45] that the string tension unlikely vanishes in the chiral limit, in contradiction to the IRconformality. To resolve the issue, it may be necessary to study the theory further by taking the possibility of realization of confinement without chiral symmetry breaking taken into account. 


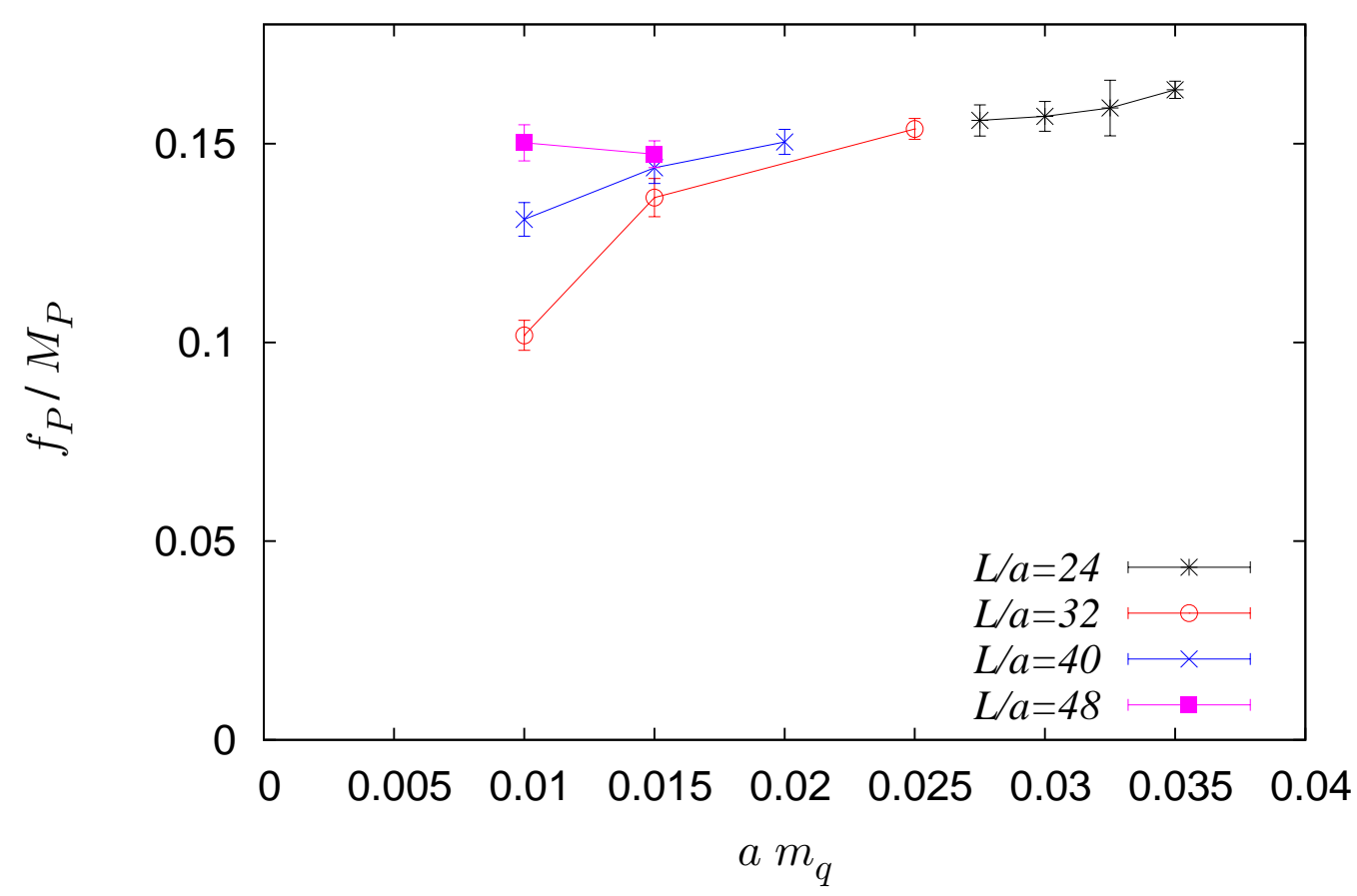

FIG. 33: Quark mass and volume dependence of the ratio $f_{P} / M_{P}$ in $\mathrm{SU}(3)_{\mathrm{C}}$ gauge theory with twelve-flavors, calculated from the data found in Ref. [23].

\section{Acknowledgments}

The numerical simulations with large lattices, $L / a \geq 16$, were carried out on the computer system $\varphi$ at Nagoya University, and the servers equipped with GPU cards at High Energy Accelerator Research Organization (KEK). This work is supported partly by JSPS Grandsin-Aid for Scientific Research 20540261, 22224003, 22740183, 23740177. M. T. is supported in part by LGS (leading graduate school) program.

\section{Appendix A: Implication on electroweak symmetry breaking}

It is often said that $\mathrm{SU}(2)_{\mathrm{C}}$ gauge theory is similar to $\mathrm{SU}(3)_{\mathrm{C}}$ gauge theory and thus no additional insight is obtained by performing separate lattice simulation. That is actually not the case, because $\mathrm{SU}(2)_{\mathrm{C}}$ gauge theory is one of $\mathrm{Sp}(2 N)_{\mathrm{C}}$ gauge theories in that its fundamental representation is pseudo-real due to the existence of a group-invariant symplectic form. As is explained below, the chiral symmetry of the system with $N_{F}$ Dirac fermions in 
the fundamental representation is thus enhanced from the usual $\mathrm{SU}\left(N_{F}\right)_{\mathrm{L}} \times \mathrm{SU}\left(N_{F}\right)_{\mathrm{R}} \times \mathrm{U}(1)_{\mathrm{B}}$ to $\mathrm{SU}\left(2 N_{F}\right)$, which is though to be broken to $\operatorname{Sp}\left(2 N_{F}\right)$ if the spontaneous breakdown occurs. Thus, it is plausible that the spectra as well as the chiral dynamics differ from those in $\mathrm{SU}(3)_{\mathrm{C}}$ gauge theories. Moreover, from the standpoint of the application of the gauge dynamics to the realization of the electroweak symmetry breaking, this fact serves the effective composite Higgs sector quite different from the $\mathrm{SU}\left(N_{C}\right)_{\mathrm{C}}$ gauge theories with $N_{C} \geq 3$. The purpose of this appendix is to summarize these basic kinematic features of $\mathrm{SU}(2)_{\mathrm{C}}$ gauge theory. We take up $\mathrm{SU}(2)_{\mathrm{C}}$ from a series of $\mathrm{Sp}(2 N)_{\mathrm{C}}$ as a structure group of the gauge theory, but the discussion in what follows persists if the symplectic form $\varepsilon_{\mathrm{C}}$ of $\mathrm{SU}(2)_{\mathrm{C}}$ is replaced with that $\mathcal{J}_{\mathrm{C}}$ of $\operatorname{Sp}(2 N)_{\mathrm{C}}$.

In the chiral representation of gamma matrices,

$\gamma^{\mu}=\left(\begin{array}{cc}0 & \left(\sigma^{\mu}\right)_{\alpha \dot{\delta}} \\ \left(\bar{\sigma}^{\mu}\right)^{\dot{\beta} \gamma} & 0\end{array}\right) \quad(\alpha, \dot{\beta}, \gamma, \dot{\delta}=1,2)$,

each of $N_{F}$ Dirac fermions, $\psi_{r, i}\left(r=1,2 ; i=1, \cdots, N_{F}\right)$, is decomposed into a pair of two-component spinors (Weyl fermions) $\left(\xi_{r, i}^{+}\right)_{\alpha}(\alpha=1,2),\left(\xi_{r, i}^{-}\right)^{\dot{\beta}}(\dot{\beta}=1,2)$, which belong to inequivalent irreducible representations of Lorentz group $\mathrm{SO}(3,1)$, as

$\psi_{r, i}=\left(\begin{array}{c}\xi_{r, i}^{+} \\ \xi_{r, i}^{-}\end{array}\right)$.

We recall that the anti-symmetric tensor $\left(\varepsilon_{\mathrm{C}}\right)_{r s}\left(r, s=1,2,\left(\varepsilon_{\mathrm{C}}\right)_{12}=1\right)$ is the symplectic form of $\mathrm{SU}(2)_{\mathrm{C}}$. Just as done for a Higgs doublet in the standard model, $\varepsilon_{r s}\left(\xi_{s, i}^{-}\right)^{*}$, where the summation over repeated indices is understood, is shown to transform in the same way as $\xi_{r, i}^{-}$with respect to $\mathrm{SU}(2)_{\mathrm{C}}$. Further multiplication of an $\mathrm{SL}(2)$-invariant anti-symmetric tensor, $\left(\varepsilon_{\mathrm{L}}\right)_{\alpha \beta}$, allows to convert $\left(\varepsilon_{\mathrm{C}}\right)_{r s}\left(\xi_{s, i}^{-}\right)^{*}$ to $\left(\xi_{r, i+N_{F}}^{+}\right)_{\alpha} \equiv\left(\varepsilon_{\mathrm{L}}\right)_{\alpha \beta}\left(\varepsilon_{\mathrm{C}}\right)_{r s}\left(\xi_{s, i}^{-\dot{\beta}}\right)^{*}$ that transforms exactly in the same way as $\xi_{r, i}^{+}$under Lorentz transformations as well as SU(2) ${ }_{C}$. A simple manipulation shows that the fermionic part of the action in the chiral limit contains $2 N_{F}$ Weyl fermions $\xi_{r, I}^{+}\left(I=1, \cdots, 2 N_{F}\right)$ on the same footing:

$S_{F}=\int d^{4} x \sum_{I=1}^{2 N_{F}} \frac{i}{2}\left(\xi_{I}^{+\dagger}\right)_{\dot{\alpha}}\left(\bar{\sigma}^{\mu}\right)^{\dot{\alpha} \beta}\left(\partial_{\mu}-i g G_{\mu}\right) \xi_{I, \beta}^{+}$.

This action is manifestly invariant under the global symmetry $G=\mathrm{SU}\left(2 N_{F}\right)$.

We suppose that the fermion-bilinear operators

$W_{I J} \equiv-\xi_{I}\left(\varepsilon_{\mathrm{C}} \otimes \varepsilon_{\mathrm{L}}\right) \xi_{J}$, 
$\left(\varepsilon_{\mathrm{C}}\right.$ and $\varepsilon_{\mathrm{L}}$ are $\mathrm{SU}(2)_{\mathrm{C}}$-invariant and Lorentz-invariant anti-symmetric tensors, respectively), which are anti-symmetric with respect to $I, J$, serve an appropriate order parameter of chiral symmetry breaking. As long as $N_{F}$ is below a certain number $N_{F}^{\text {crtl }}$, the non-perturbative dynamics are expected to give them non-zero vacuum expectation values (VEVs) of the form $\left\langle W_{I J}\right\rangle=\frac{1}{2} \Sigma \mathcal{J}_{I J}$

where $\mathcal{J}$ denotes the symplectic form of the subgroup $H=\operatorname{Sp}\left(2 N_{F}\right)$ of $G=\operatorname{SU}\left(2 N_{F}\right)$ and $\Sigma$ can be taken to be real, implying that the chiral symmetry $G$ is spontaneously broken down to $H$. In the basis such that $\mathcal{J}$ takes the form

$\mathcal{J}_{I J}=-\delta_{I+N_{F}, J}+\delta_{I, J+N_{F}}$.

VEVs in Eq. (A5) imply that

$\left\langle\bar{\psi}^{i} \psi_{j}\right\rangle=-\Sigma \delta_{j}^{i}$

which is the same form as in QCD whose structure group is $\mathrm{SU}(3)_{\mathrm{C}}$. From this fact, one anticipates the pattern of breaking $\mathrm{SU}\left(2 N_{F}\right) \rightarrow \mathrm{Sp}\left(2 N_{F}\right)$.

Just as in the same manner, the degenerate Dirac mass terms, $m \sum_{i=1}^{N_{F}} \bar{\psi}^{i} \psi_{i}$, can be seen to be invariant under $\operatorname{Sp}\left(2 N_{F}\right)$. Thus, we expect that the lattice actions and techniques developed for three-color QCD serve a way to examine whether the breaking pattern $\mathrm{SU}\left(2 N_{F}\right) \rightarrow \mathrm{Sp}\left(2 N_{F}\right)$ occurs by taking the vanishing "external source" limit, $m \rightarrow 0$.

Next, we observe the implication of the application of the dynamics of $\mathrm{SU}(2)_{\mathrm{C}}$ gauge theories to the electroweak symmetry breaking. This needs specification of assignment of charges (representations) under $G_{\mathrm{EW}}=\mathrm{SU}(2)_{\mathrm{L}} \times \mathrm{U}(1)_{\mathrm{Y}}$, i.e. the way of embedding of $G_{\mathrm{EW}}$ into $G=\mathrm{SU}\left(2 N_{F}\right)$. Our dynamical assumption is that $G \rightarrow H=\operatorname{Sp}\left(2 N_{F}\right)$ with (A5) at the zeroth order of the interactions other than $\mathrm{SU}(2)_{\mathrm{C}}$, i.e. $G_{\mathrm{EW}}$-gauge interactions, "real" QCD, and the interactions from the structure responsible to generating masses of quarks and leptons (extended technicolor, ETC) [3]. Even though $H$ has degeneracy $G / H$ in $G$ at the zeroth order, the position will be fixed so that the energy due to radiative corrections of those interactions is minimized [61, 62]. The unbroken gauge symmetry is $H \cap G_{\mathrm{EW}}$ with $H$ determined as such, and must be the symmetry of electromagnetism, $U(1)_{\mathrm{em}}$.

Since only relative position in $G$ matters, vacuum alignment can be asked by fixing $H$ in $G$ so that the chiral condensates take the form (A5) with $\mathcal{J}$ in Eq. (A6), and questioning 
which position of $G_{\mathrm{EW}}$ in $G$ minimize the vacuum energy. Denoting a pair of an irreducible representation $\mathbf{r}$ of $\mathrm{SU}(2)_{L}$ and the charge $\frac{Y}{2}$ with respect to $\mathrm{U}(1)_{\mathrm{Y}}$ by $\left(\mathbf{r}, \frac{\mathrm{Y}}{2}\right)$, we start with $N_{F}=2$ and the following simple example

$$
\left(\begin{array}{l}
\xi_{1} \\
\xi_{2}
\end{array}\right) \Leftrightarrow(\mathbf{2}, 0), \quad \xi_{3} \Leftrightarrow\left(0,-\frac{1}{2}\right), \quad \xi_{4} \Leftrightarrow\left(0,+\frac{1}{2}\right) .
$$

We can see that the condensates of the form (A5) is invariant under the subgroup $\mathrm{U}(1)_{\mathrm{em}}$ but not under the whole $G_{\mathrm{EW}}$. However, there is another embedding way of $G_{\mathrm{EW}}$ into $G$ as follows:

$$
\left(\begin{array}{c}
\xi_{1} \\
\xi_{3}
\end{array}\right) \Leftrightarrow\left(\frac{1}{2}, 0\right), \quad \xi_{2} \Leftrightarrow\left(0,-\frac{1}{2}\right), \quad \xi_{4} \Leftrightarrow\left(0,+\frac{1}{2}\right) .
$$

In this case, the condensates (A5) are invariant under the whole $G_{\mathrm{EW}}$ so that $G_{\mathrm{EW}}$ remains unbroken.

The above example highlights the essence of a property of the "effective Higgs sector" of $\mathrm{Sp}(2 N)_{\mathrm{C}}$ gauge theories. In order to break $\mathrm{SU}(2)_{\mathrm{L}}$, there must be at least one $\mathrm{SU}(2)_{\mathrm{L}^{-}}$ nonsinglet $\xi_{I}(I=1, \cdots, n)$ with $n \geq 2$. The chiral condensates $W_{I J} \equiv-\xi_{I}\left(\mathcal{J}_{\mathrm{C}} \otimes \varepsilon_{\mathrm{L}}\right) \xi_{J}$ defined as in Eq. (A5) will contain the multiplet transforming as $\mathbf{n}$. An even $n$, such as 2, may be suitable to inducing the desirable breaking pattern $G_{\mathrm{EW}} \rightarrow \mathrm{U}(1)_{\mathrm{em}}$. Since the chiral condensates are anti-symmetric with respect to flavor indices, they inevitably contain an

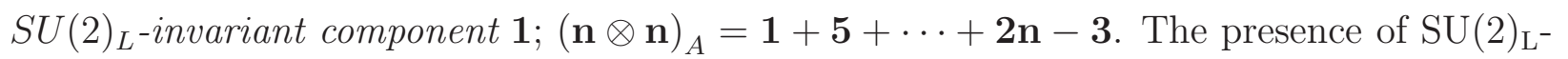
singlet composite field is one characteristic property of the effective composite Higgs sector of $\mathrm{Sp}(2 N)_{\mathrm{C}}$ gauge theory. If there are $\mathrm{SU}(2)_{\mathrm{L}}$-multiplet with $n$ equal to or greater than 4 , the VEVs of the composite fields belonging to the multiplet of odd dimension $\geq 5$ can shift the $\rho$ parameter significantly from 1 , unless the dynamics of $\operatorname{Sp}(2 N)_{\text {C }}$ gauge theory function to suppress them.

A similar consideration brings out the basic feature of the "effective Higgs sector" of $\mathrm{SO}(2 N)_{\mathrm{C}}$ gauge theories. The chiral condensates $N_{I J} \equiv-\xi_{I} \varepsilon_{L} \xi_{J}$ are symmetric with respect to $I, J$. For a given $n, N_{I J}$ contains $(\mathbf{n} \otimes \mathbf{n})_{S}=\mathbf{3}+\mathbf{7}+\cdots+\mathbf{2 n}-\mathbf{1}$. Therefore, the effective composite Higgs sector of $\mathrm{SO}(2 N)_{\mathrm{C}}$ gauge theory inevitably contains the $\mathrm{SU}(2)_{\mathrm{L}^{-}}$ triplet composite Higgs field, as mentioned above, the VEV of which yields inconsistency 
with the experimental constraint.

[1] L. Susskind, "Dynamics of Spontaneous Symmetry Breaking in the Weinberg-Salam Theory," Phys.Rev. D20 (1979) 2619-2625.

[2] S. Weinberg, "Implications of Dynamical Symmetry Breaking," Phys.Rev. D13 (1976) 974-996.

[3] S. Dimopoulos and L. Susskind, "Mass Without Scalars," Nucl.Phys. B155 (1979) 237-252.

[4] S. Raby, S. Dimopoulos, and L. Susskind, "Tumbling Gauge Theories," Nucl.Phys. B169 (1980) 373.

[5] E. Farhi and L. Susskind, "Technicolor," Phys.Rept. 74 (1981) 277.

[6] M. E. Peskin and T. Takeuchi, "A New constraint on a strongly interacting Higgs sector," Phys.Rev.Lett. 65 (1990) 964-967.

[7] M. E. Peskin and T. Takeuchi, "Estimation of oblique electroweak corrections," Phys.Rev. D46 (1992) 381-409.

[8] B. Holdom, "Raising the Sideways Scale," Phys.Rev. D24 (1981) 1441.

[9] K. Yamawaki, M. Bando, and K. Matumoto, "Scale Invariant Technicolor Model and a Technidilaton," Phys.Rev.Lett. 56 (1986) 1335.

[10] T. Akiba and T. Yanagida, "Hierarchic Chiral Condensate," Phys.Lett. B169 (1986) 432.

[11] T. W. Appelquist, D. Karabali, and L. Wijewardhana, "Chiral Hierarchies and the Flavor Changing Neutral Current Problem in Technicolor," Phys.Rev.Lett. 57 (1986) 957.

[12] JLQCD Collaboration, E. Shintani et al., "S-parameter and pseudo-Nambu-Goldstone boson mass from lattice QCD," Phys.Rev.Lett. 101 (2008) 242001, arXiv:0806.4222 [hep-lat],

[13] RBC, UKQCD Collaboration, P. A. Boyle, L. Del Debbio, J. Wennekers, and J. M. Zanotti, "The S Parameter in QCD from Domain Wall Fermions," Phys.Rev. D81 (2010) 014504, arXiv:0909.4931 [hep-lat].

[14] LSD Collaboration, T. Appelquist et al., "Parity Doubling and the S Parameter Below the Conformal Window," Phys.Rev.Lett. 106 (2011) 231601, arXiv:1009.5967 [hep-ph].

[15] T. Appelquist, G. T. Fleming, and E. T. Neil, "Lattice study of the conformal window in QCD-like theories," Phys.Rev.Lett. 100 (2008) 171607, arXiv:0712.0609 [hep-ph]. 
Erratum: ibid., 102, 149902 (2009).

[16] T. Appelquist, G. T. Fleming, and E. T. Neil, "Lattice Study of Conformal Behavior in SU(3) Yang-Mills Theories," Phys.Rev. D79 (2009) 076010, arXiv:0901.3766 [hep-ph].

[17] M. Luscher, R. Narayanan, P. Weisz, and U. Wolff, "The Schrodinger functional: A Renormalizable probe for nonAbelian gauge theories," Nucl.Phys. B384 (1992) 168-228, arXiv:hep-lat/9207009 [hep-lat].

[18] M. Luscher, R. Sommer, U. Wolff, and P. Weisz, "Computation of the running coupling in the SU(2) Yang-Mills theory," Nucl.Phys. B389 (1993) 247-264, arXiv:hep-lat/9207010 [hep-lat].

[19] S. Sint, "On the Schrodinger functional in QCD," Nucl.Phys. B421 (1994) 135-158, arXiv:hep-lat/9312079 [hep-lat].

[20] Y. Iwasaki, K. Kanaya, S. Kaya, S. Sakai, and T. Yoshie, "Phase structure of lattice QCD for general number of flavors," Phys.Rev. D69 (2004) 014507 , arXiv:hep-lat/0309159 [hep-lat].

[21] T. DeGrand, Y. Shamir, and B. Svetitsky, "Phase structure of SU(3) gauge theory with two flavors of symmetric-representation fermions," Phys.Rev. D79 (2009) 034501, arXiv:0812.1427 [hep-lat].

[22] T. DeGrand, "Finite-size scaling tests for SU(3) lattice gauge theory with color sextet fermions," Phys.Rev. D80 (2009) 114507, arXiv:0910.3072 [hep-lat].

[23] Z. Fodor, K. Holland, J. Kuti, D. Nogradi, and C. Schroeder, "Twelve massless flavors and three colors below the conformal window," Phys.Lett. B703 (2011) 348-358, arXiv:1104.3124 [hep-lat].

[24] A. Hasenfratz, "Investigating the critical properties of beyond-QCD theories using Monte Carlo Renormalization Group matching," Phys.Rev. D80 (2009) 034505. arXiv:0907.0919 [hep-lat].

[25] A. Hasenfratz, "Conformal or Walking? Monte Carlo renormalization group studies of SU(3) gauge models with fundamental fermions," Phys.Rev. D82 (2010) 014506, arXiv:1004.1004 [hep-lat].

[26] A. Hasenfratz, "Infrared fixed point of the 12-fermion SU(3) gauge model based on 2-lattice MCRG matching," Phys.Rev.Lett. 108 (2012) 061601, arXiv:1106.5293 [hep-lat].

[27] L. Del Debbio, B. Lucini, A. Patella, C. Pica, and A. Rago, "Mesonic spectroscopy of 
Minimal Walking Technicolor," Phys.Rev. D82 (2010) 014509,

arXiv:1004.3197 [hep-lat].

[28] L. Del Debbio and R. Zwicky, "Hyperscaling relations in mass-deformed conformal gauge theories," Phys.Rev. D82 (2010) 014502, arXiv:1005.2371 [hep-ph].

[29] E. Bilgici, A. Flachi, E. Itou, M. Kurachi, C.-J. D. Lin, et al., "Search for the IR fixed point in the twisted Polyakov loop scheme," PoS LAT2009 (2009) 063,

arXiv:0910.4196 [hep-lat].

[30] E. Itou, "Properties of the twisted Polyakov loop coupling and the infrared fixed point in the SU(3) gauge theory," arXiv:1212.1353 [hep-lat].

[31] K.-I. Ishikawa, Y. Iwasaki, Y. Nakayama, and T. Yoshie, "Conformal Theories with IR cutoff," arXiv:1301.4785 [hep-lat].

[32] T. Appelquist et al., "Approaching Conformality with Ten Flavors," arXiv:1204.6000 [hep-ph].

[33] M. Hayakawa, K.-I. Ishikawa, Y. Osaki, S. Takeda, S. Uno, and N. Yamada, "Running coupling constant of ten-flavor QCD with the Schródinger functional method," Phys.Rev. D83 (2011) 074509, arXiv:1011.2577 [hep-lat].

[34] J. Kuti, J. Polonyi, and K. Szlachanyi, "Monte Carlo Study of SU(2) Gauge Theory at Finite Temperature," Phys.Lett. B98 (1981) 199.

[35] J. Engels, F. Karsch, H. Satz, and I. Montvay, "High Temperature SU(2) Gluon Matter on the Lattice," Phys.Lett. B101 (1981) 89.

[36] B. Svetitsky and L. G. Yaffe, "Critical Behavior at Finite Temperature Confinement Transitions," Nucl.Phys. B210 (1982) 423.

[37] J. Kogut and D. Sinclair, "SU(2) AND SU(3) LATTICE GAUGE THEORIES WITH MANY FERMIONS," Nucl.Phys. B295 (1988) 465.

[38] F. Bursa, L. Del Debbio, L. Keegan, C. Pica, and T. Pickup, "Mass anomalous dimension in SU(2) with six fundamental fermions," Phys.Lett. B696 (2011) 374-379, arXiv:1007.3067 [hep-ph].

[39] H. Ohki et al., "Study of the scaling properties in SU(2) gauge theory with eight flavors," PoS LATTICE2010 (2010) 066, arXiv:1011.0373 [hep-lat].

[40] T. Karavirta, J. Rantaharju, K. Rummukainen, and K. Tuominen, "Determining the conformal window: $\mathrm{SU}(2)$ gauge theory with $N_{f}=4,6$ and 10 fermion flavours," 
JHEP 1205 (2012) 003, arXiv:1111.4104 [hep-lat].

[41] LSD Collaboration, G. Voronov, "Lattice study of the extent of the conformal window in two-color Yang-Mills theory," PoS LATTICE2011 (2011) 093.

[42] G. Voronov, "Two-Color Schrödinger Functional with Six-Flavors of Stout-Smeared Wilson Fermions," PoS LAT2012 (2012) 039.

[43] T. van Ritbergen, J. Vermaseren, and S. Larin, "The Four loop beta function in quantum chromodynamics," Phys.Lett. B400 (1997) 379-384, arXiv:hep-ph/9701390 [hep-ph].

[44] M. Hayakawa, K. I. Ishikawa, S. Takeda, and N. Yamada, "Running coupling constant and mass anomalous dimension of six-flavor SU(2) gauge theory," arXiv:1307.6997 [hep-lat].

[45] M. Hayakawa, K.-I. Ishikawa, Y. Osaki, S. Takeda, and N. Yamada, "Lattice study on two-color QCD with six flavors of dynamical quarks," PoS LATTICE2012 (2012) 040, arXiv:1210.4985 [hep-lat].

[46] J. Gasser and H. Leutwyler, "Light Quarks at Low Temperatures," Phys.Lett. B184 (1987) 83.

[47] M. Luscher, "On a relation between finite size effects and elastric scattering process," NATO ASI Series 115 (1984) 451-472.

[48] M. Luscher, "Volume Dependence of the Energy Spectrum in Massive Quantum Field Theories. 1. Stable Particle States," Commun.Math.Phys. 104 (1986) 177.

[49] Y. Koma and M. Koma, "On the finite size mass shift formula for stable particles," Nucl.Phys. B713 (2005) 575-597, arXiv:hep-lat/0406034 [hep-lat].

[50] S. Duane, A. Kennedy, B. Pendleton, and D. Roweth, "Hybrid Monte Carlo," Phys.Lett. B195 (1987) 216-222.

[51] I. P. Omelyan, I. M. Mryglod, and R. Folk, "Symplectic analytically integrable decomposition algorithms: classification, derivation, and application to molecular dynamics, quantum and celestial mechanics simulations," Comp.Phys.Commun. 151 (2003) 272.

[52] M. Hayakawa, K.-I. Ishikawa, Y. Osaki, S. Takeda, S. Uno, and N. Yamada, "Improving many flavor QCD simulations using multiple GPUs," PoS LATTICE2010 (2010) 325, arXiv:1009.5169 [hep-lat].

[53] UKQCD Collaboration, C. Allton et al., "Gauge invariant smearing and matrix correlators using Wilson fermions at Beta = 6.2," Phys.Rev. D47 (1993) 5128-5137, arXiv:hep-lat/9303009 [hep-lat]. 
[54] M. Bochicchio, L. Maiani, G. Martinelli, G. C. Rossi, and M. Testa, "Chiral Symmetry on the Lattice with Wilson Fermions," Nucl.Phys. B262 (1985) 331.

[55] K.-I. Nagai, G. Carrillo-Ruiz, G. Koleva, and R. Lewis, "Exploration of SU(N(c)) gauge theory with many Wilson fermions at strong coupling," Phys.Rev. D80 (2009) 074508, arXiv:0908.0166 [hep-lat].

[56] WHOT-QCD Collaboration Collaboration, T. Umeda et al., "Thermodynamics in $2+1$ flavor QCD with improved Wilson quarks by the fixed scale approach," PoS LATTICE2012 (2012) 074, arXiv:1212.1215 [hep-lat].

[57] G. Colangelo, S. Durr, and C. Haefeli, "Finite volume effects for meson masses and decay constants," Nucl.Phys. B721 (2005) 136-174, arXiv:hep-lat/0503014 [hep-lat].

[58] S. Aoki and H. Fukaya, "Interpolation between the epsilon and p regimes," Phys.Rev. D84 (2011) 014501, arXiv:1105.1606 [hep-lat].

[59] J. Gasser and H. Leutwyler, "Chiral Perturbation Theory: Expansions in the Mass of the Strange Quark," Nucl.Phys. B250 (1985) 465.

[60] F. Bursa, L. Del Debbio, D. Henty, E. Kerrane, B. Lucini, et al., "Improved Lattice Spectroscopy of Minimal Walking Technicolor," Phys.Rev. D84 (2011) 034506, arXiv:1104.4301 [hep-lat].

[61] M. E. Peskin, "The Alignment of the Vacuum in Theories of Technicolor," Nucl.Phys. B175 (1980) 197-233.

[62] J. Preskill, "Subgroup Alignment in Hypercolor Theories," Nucl.Phys. B177 (1981) 21-59. 